\title{
Characteristics and outcomes in patients with atrial fibrillation receiving direct oral anticoagulants in off-label doses
}

\author{
Alexandros Briasoulis ${ }^{1,2^{*}}$ (D), Yubo Gao ${ }^{3}$, Chakradhari Inampudi ${ }^{1}$, Paulino Alvarez ${ }^{1}$, Rabea Asleh ${ }^{1}$, \\ Elizabeth Chrischilles ${ }^{4}$, Enrique C. Leira ${ }^{4,5}$ and Mary Vaughan-Sarrazin ${ }^{3,6^{*}}$
}

\begin{abstract}
Background: We evaluated adherence to dosing criteria for patients with atrial fibrillation (AF) taking dabigatran or rivaroxaban and the impact of off-label dosing on thromboembolic and bleeding risk.

Methods: We used data for a retrospective cohort from a large U.S. health plan for Medicare beneficiaries age $>=$ 65 years with AF who initiated dabigatran or rivaroxaban during 2010-2016. Stroke and major bleeding were quantified in patients who were eligible for low dose but received standard dose, and in patients who were eligible for standard dose but received low dose.

Results: We identified 8035 and 19,712 patients who initiated dabigatran or rivaroxaban, respectively. Overall, 1401 (17.4\%) and 7820 (39.7\%) patients who received dabigatran and rivaroxaban met criteria for low dose, respectively. Of those, 959 (68.5\%) and 3904 (49.9\%) received standard dose. In contrast, 1013 (15.3\%) and 2551 (21.5\%) of patients eligible for standard dose dabigatran and rivaroxaban received low dose. Mean follow-up for patients eligible for low and standard dose dabigatran and rivaroxaban were 13.9, 15.1, 10.1, and 12.3 months, respectively. In unadjusted analyses, patients eligible for low or standard dose dabigatran and rivaroxaban but receiving off-label dose, had no differences in the rates of ischemic stroke. Among patients who met criteria for standard dose direct oral anticoagulants $(\mathrm{DOAC})$, use of low dose was associated with significantly higher risk of any major bleeding (Dabigatran: $\mathrm{HR}=1.44 ; 95 \% \mathrm{Cl} 1.14-1.8, P=0.002$, Rivaroxaban HR 1.34, 95\% Cl 1.11-1.6, $P=0.002$ ) and gastrointestinal bleeding (Dabigatran: $\mathrm{HR}=1.48 ; 95 \% \mathrm{Cl} 1.08-2, P=0.016$ ). In patients who met criteria for low dose DOACs, there was lower risk of major bleeding (Dabigatran: $\mathrm{HR}=0.59 ; 95 \% \mathrm{Cl} 0.43-0.8, P<0.001$ ), gastrointestinal (Rivaroxaban: HR 0.79; 95\% Cl 0.64-0.98, $P=0.03$ ) and intracranial bleeding (Dabigatran: $\mathrm{HR}=0.33 ; 95 \% \mathrm{Cl} 0.12-0.9$, $P=0.001)$ with standard dosing. After propensity matching, use of off-label doses was not associated with stroke, major, gastrointestinal or intracranial bleeding for either dabigatran or rivaroxaban.
\end{abstract}

Conclusions: While a significant number of patients receive higher or lower dose of dabigatran and rivaroxaban than recommended, we found no evidence of significant impact on thromboembolic or hemorrhagic outcomes.

Keywords: Atrial fibrillation, Stroke, Bleeding, Direct oral anticoagulants

\footnotetext{
*Correspondence: alexbriasoulis@gmail.com; mary-vaughansarrazin@uiowa.edu

'Division of Cardiovascular Diseases, Section of Heart Failure and Transplant, University of lowa College of Medicine, lowa City, IA, USA

${ }^{3}$ Division of General Medicine, University of lowa College of Medicine, lowa City, IA, USA

Full list of author information is available at the end of the article
}

(c) The Author(s). 2020 Open Access This article is distributed under the terms of the Creative Commons Attribution 4.0 International License (http://creativecommons.org/licenses/by/4.0/), which permits unrestricted use, distribution, and reproduction in any medium, provided you give appropriate credit to the original author(s) and the source, provide a link to the Creative Commons license, and indicate if changes were made. The Creative Commons Public Domain Dedication waiver (http://creativecommons.org/publicdomain/zero/1.0/) applies to the data made available in this article, unless otherwise stated. 


\section{Background}

Patients with Atrial fibrillation (AF) have a higher risk for stroke or systemic embolism, death and disability [1]. Oral anticoagulants, either vitamin $\mathrm{K}$ antagonist (VKA) or direct oral anticoagulants (DOACs) reduce that thromboembolic risk by about two-thirds irrespective of baseline risk [2]. However, the use of anticoagulation is associated with increased risk of bleeding, with intracranial hemorrhage $(\mathrm{ICH})$ being the most serious bleeding complication [3]. Randomized controlled trials (RCT) of DOACs [Dabigatran, Rivaroxaban, Apixaban, and Edoxaban] have demonstrated similar protection against ischemic stroke but lower rates of ICH compared with VKAs [4-7].

The RCTs of DOACs in AF patients used dose adjustments based on patient characteristics such age, weight, renal function and the use of concomitant medications. A reduced dose of $75 \mathrm{mg}$ twice daily of dabigatran is recommended to decrease bleeding risk in patients with creatinine clearance $(\mathrm{CrCl}) 15-30 \mathrm{~mL} /$ minute, or co- administration of a strong P-glycoprotein [P-gp] inhibitor (e.g., dronedarone) in patients with $\mathrm{CrCl} 30-50 \mathrm{~mL} / \mathrm{mi}-$ nute $[8,9]$. With regards to rivaroxaban, a dose reduction to $15 \mathrm{mg}$ daily is recommended in patients with $\mathrm{CrCl} 15-50 \mathrm{~mL} /$ minute, and concomitant use of a dual P-gp and cytochrome-3A4 [P-gp-Cyp3A4] inhibitor should be avoided to prevent potential increased rivaroxaban concentration [10]. Administration of lower apixaban dose $2.5 \mathrm{mg}$ twice daily is indicated if 2 of the following 3 criteria are met: age $>80$ years, weight $<$ than $60 \mathrm{~kg}$, and serum Creatinine $>1.5 \mathrm{mg} / \mathrm{dl}$ [11].

Since the use of DOACs became widespread, deviations from the recommended dosing are not infrequent $[12,13]$. Analysis of 5738 patients treated with DOACs from the ORBITA-AF II registry showed that $9.4 \%$ of patients were under-dosed and 3.4\% were overdosed. Overdosing was associated with significantly increased all-cause mortality whereas under-dosing was associated with increased cardiovascular hospitalizations [14]. A subsequent analysis of 7925 AF patients treated with DOACs from the same registry showed that $16 \%$ of patients were on reduced doses with many of these doses adjustments (57\%) not following the recommended doses [15]. Nevertheless, after risk- adjustment, the use of lower-than-recommended dose resulted in similar thromboembolic and bleeding risk compared to appropriately dosed DOAC use [15].

We hypothesized that a sizeable number of DOAC prescriptions do not adhere to the Food and Drug Administration (FDA) dosing criteria and may increase thromboembolic and bleeding events. The purpose of our study was to: 1) examine characteristics and predictors of low dose use among patients who meet FDA criteria for standard dose, or standard dose use among patients who meet FDA criteria for low dose, among patients who initiate dabigatran and rivaroxaban, ii) compare the risk of ischemic stroke and bleeding events in patients receiving off-label low dose or off-label standard dose to patients receiving FDA-recommended doses in a community-based sample of elderly Medicare beneficiaries with AF enrolled in a large U.S. health plan.

\section{Methods}

\section{Data source}

We designed a new user retrospective cohort study using data for Medicare beneficiaries enrolled in a large U.S. health plan with prescription drug coverage. Medical (inpatient visit, outpatient physician visits) and pharmacy claims with detailed prescription fill information from October 1, 2010 through December 31, 2016 were analyzed. In addition, the data also includes laboratory test results (such as serum creatinine) for Medicare beneficiaries enrolled in managed care plans. The study was nonhuman subject research by the University of Iowa institutional review board because it involved analysis of an existing database that was fully de-identified.

\section{Patient population}

We analyzed claims for Medicare beneficiaries age $>=65$ years with newly diagnosed atrial fibrillation (AF) between 2010 and 2016 (dabigatran was approved by FDA in October 2010 followed by rivaroxaban approval in November 2011). We identified patients who initiated dabigatran $150 \mathrm{mg}$ twice daily (standard dose) or $75 \mathrm{mg}$ twice daily (low dose), or rivaroxaban $20 \mathrm{mg}$ daily (standard dose) or $15 \mathrm{mg}$ daily. We did not include patients receiving apixaban as information to assess dosing criteria such as patient weight was not available in our data, and we did not include patients receiving edoxaban due to relatively low use of this drug during our time frame. Patients were categorized into mutually exclusive groups according to the first DOAC and DOAC dose received.

Patients were excluded if they did not have a diagnosis of AF during the 12 months prior to initiating the DOAC, where AF was defined as International Classification of Diseases-Ninth Revision-Clinical Modification [ICD-9-CM] code 427.31 or ICD-Tenth Revision [ICD10] code I48.0, I48.1, I48.2, I48.3, I48.4, or I48.91, as primary or secondary diagnosis. Additionally, we excluded patients who: i) were younger than 65 years at the time of diagnosis, ii) had a diagnosis indicating pulmonary embolism or deep vein thrombosis within 8 weeks prior to initiating the DOAC, iii) underwent hip surgery within 6 weeks prior to initiating the DOAC, or iv) were not enrolled in the health care plan for at least 1 year prior to initiating the DOAC.

Patients who initiated dabigatran or rivaroxaban were categorized to approximate dosing criteria, using the 
most recent estimated glomerular filtration rate (eGFR) available prior to initiating the DOAC as a proxy for creatinine clearance. eGFR was calculated based on Modification of Diet in Renal Disease (MDRD) Study equation or Chronic Kidney Disease Epidemiology Collaboration (CKD-EPI) equation $[16,17]$. Patients who received dabigatran were deemed to meet criteria for low dose if they had severe renal disease (defined as eGFR $<30 \mathrm{~mL} / \mathrm{mi}$ nute/ $1.73 \mathrm{~m} 2$ ) or had moderate renal disease and concurrent use of a p-gp inhibitor (where moderate renal disease was defined as eGFR $30-50 \mathrm{~mL} /$ minute $/ 1.73 \mathrm{~m} 2$ and p-gp inhibitors included dronedarone, cyclosporine, itraconazole, tacrolimus, ketoconazole). Patients who received rivaroxaban were deemed to meet criteria for low dose if they had eGFR $<50$ or concomitant use of a dual P-gpCyp3A4 inhibitor (including ketoconazole, fluconazole, itraconazole, cobicistat, conivaptan, indinavir, voriconazole, posaconazole, nefazodone $\mathrm{HCL}$, ritonavir, saquinavir, telithromycin). Patients with no valid GFR for assessing renal function were excluded. Among patients eligible for low doses of dabigatran or rivaroxaban, 87.4 and $86.5 \%$ had available eGFR whereas among those eligible for standard dose dabigatran or rivaroxaban, 54.9 and 56\% had available eGFR respectively. We performed sensitivity analysis for DOAC dose adjustments based on eGFR only, excluding drug interactions.

\section{Covariates}

Data on patient-level characteristics such as demographics, comorbid conditions, concurrent medication use, and prior health services utilization were extracted from health care plan enrollment data and inpatient, outpatient, and physician claims. Comorbid diseases were identified by ICD-9-CM/ICD-10 diagnoses on claims during the 12 months preceding the date of first DOAC fill. We used the Charlson Comorbidity Index to estimate patients' overall comorbidity status [18]. We also identified all conditions in the CHA2DS2-VASc stroke risk score (congestive heart failure diagnosis, female sex, hypertension diagnosis, diabetes, age, prior stroke or transient ischemic attack, and vascular disease diagnosis). History of major bleeding was defined as any prior major bleeding, gastrointestinal bleeding, intracranial hemorrhage, or prior receipt of transfusion. Additional conditions included liver disease, alcohol abuse, obesity, chronic obstructive pulmonary disease, peripheral vascular disease, pulmonary circulation disease, heart valve disease, history of coronary revascularization, history of implantable cardiac device, and prior pulmonary embolism or deep vein thrombosis. We also extracted data on medication use at the time of DOAC initiation (p-glycoprotein inhibitors, cytochrome 3A4 inhibitors, insulin, statins, beta blockers, angiotensin-converting-enzyme (ACE) inhibitors, angiotensin-receptor blockers (ARBs), calcium channel blockers, prescription antiplatelets (e.g., clopidogrel), proton pump inhibitors and non-steroidal anti-inflammatory drugs. (A list of included drugs and ICD9/ICD10 codes for comorbid conditions is provided in Additional file 1: Table S1). Medications were considered concomitant if the patient had supply within 90 days from the DOAC prescription. We also identified patients with a history of warfarin use prior to initiating the DOAC.

\section{Endpoints}

We selected the following clinical endpoints: i) ischemic stroke, ii) any major bleeding, iii) gastrointestinal bleeding (GI), iv) intracranial bleeding based on the primary ICD-9$\mathrm{CM} / \mathrm{ICD}-10$ diagnosis on inpatient claims for acute care stays (definitions provided in Additional file 1: Table S1). We also examined drug discontinuation, as defined by the date of last fill for the original DOAC and dose. Patients were followed from the date of the initial DOAC prescription until December 31, 2016 or lapse of health plan enrollment (due to death or other reason), or cessation of the initial DOAC dose.

\section{Statistical analysis}

Analyses were conducted separately for patients who initiated dabigatran and rivaroxaban, and by dose eligibility. For example, among patients who received dabigatran and met criteria for low dose dabigatran, we compared characteristics and outcomes among patients who received standard dose in contrast to dosing criteria vs. those who received the recommended low dose. Similarly, among patients who received dabigatran and met criteria for standard dose, we compared patients who received low dose vs. those who received the recommended standard dose. We compared demographic characteristics, comorbid diseases, and concurrent medication use among patients on different doses using the chi-square test.

We examined rates of ischemic stroke, any major bleeding, GI hemorrhage, and intracranial hemorrhage per patient-year of follow-up in the full sample and in propensity matched patients. Specifically, we performed 2-way nearest-neighbor propensity-matching to create groups of patients receiving low dose or standard dose that were balanced with respect to observed patient characteristics. Propensity matching was conducted separately for patients qualifying for low or standard dose, and for patients on dabigatran or rivaroxaban (i.e., four separate propensity matched samples). We assessed covariate balance in propensity-matched samples using standardized differences between patients receiving low or standard dose, where differences less than $10 \%$ indicate satisfactory balance. Because standardized differences remained greater than $10 \%$ for a small number of 
covariates, we further adjusted for unbalanced covariates if they were related to the outcome of interest using Cox proportional hazards regression models. We then calculated rates of each endpoint per patient year of followup in unmatched and propensity-matched samples. Statistical significance was assessed using Cox proportional hazards regression models that censored for end of follow-up (December 31, 2016), medication cessation, or disenrollment from the managed care plan for any reason. Results were reported as hazard ratios (HR) with 95\% confidence intervals (CI) for each drug and recommended dose, where the HR reflects the outcome rate for off-label dosing relative to FDA-recommended dose. Finally, one sensitivity analysis was performed in which drug dosing criteria was based on GFR measurements only, without considering pharmacologic interactions reflected in FDA criteria.

All analyses were conducted using SAS with 2-tailed level of significance set at 0.05 .

\section{Results}

\section{Patient characteristic}

We identified 8035 patients with valid GFR measurements including 6580 on standard dabigatran dose and 1455 patients on low dose (18.1\%); 19,712 patients were rivaroxaban including 13,245 on standard dose rivaroxaban and 6467 on low dose (32.8\%). We identified significant differences in baseline characteristics between different does of dabigatran and rivaroxaban before propensity matching (Tables 1 and 2). Among dabigatran patients, those taking low dose were more likely to be over 75 years $(P<0.001)$, female $(P=0.01)$, have CKD III or more advanced $(P<0.001)$ and higher burden of comorbidities as indicated by higher Charlson comorbidity index (low dose: $5.52 \pm 3.75$ vs. high dose: $4.17 \pm 3.47$, $P<0.001)$. Among rivaroxaban patients, low dose was more frequently prescribed to patients over 75 years $(P<0.001)$, females $(P=0.03)$, patients with CKD III or more advanced $(P<0.001)$ and patients with higher comorbidity burden (low dose: $5.05 \pm 3.65$ vs. high dose: $3.71 \pm 3.34, P<0.001$ ).

As shown on Tables 3, 4, 5 and 6, 1401 (17.4\%) and $7820(39.7 \%)$ patients who received dabigatran and rivaroxaban met criteria for low dose, respectively. Of those, $959(68.5 \%)$ and $3904(49.9 \%)$ received standard dose of dabigatran and rivaroxaban respectively. In contrast, $1013(15.3 \%)$ and 2551 (21.5\%) of patients eligible for standard dose dabigatran and rivaroxaban received low dose. Patients older than 75 years, females, African Americans, and patients with history of major bleeding or heart failure were more likely to receive lower than recommended dose of dabigatran or rivaroxaban (Tables 3, 4, 5 and 6). Conversely, patients eligible for low dose dabigatran or rivaroxaban that received standard dose were more likely younger with lower rates of advanced $C K D$.

Mean follow-up for patients eligible for low dose dabigatran, standard dose dabigatran, low dose rivaroxaban, and standard dose rivaroxaban were 13.9, 15.1, 10.1, and 12.3 months, respectively.

\section{Outcomes \\ Stroke}

The absolute event rates and event rates/year for ischemic stroke in each dosing category are presented in Table 7. Before adjustment for patient characteristics or propensity-match analysis, use of low dose dabigatran among patients eligible for standard dose dabigatran did not affect ischemic stroke risk. Among those eligible for standard dose rivaroxaban but receiving low dose, no significantly different risk of ischemic stroke was found. Among patients eligible for low dose dabigatran who received standard dose, we did not identify any relationship to ischemic stroke risk (Table 7). Also, among patients eligible for low dose rivaroxaban, use of standard dose rivaroxaban was not associated with increased risk of ischemic stroke. After propensity matching, we found no difference in risk of ischemic stroke in 732 patients eligible for low dose dabigatran who received low dose compared to 732 matched patients eligible for low dose dabigatran who received standard dose, or among propensity-matched patients eligible for standard dose dabigatran who received either standard dose $(n=1960)$ or low dose $(n=1960)$. Similarly, analysis of propensity matched samples of patients eligible for low dose rivaroxaban (5328 on low dose and 5328 on standard dose) or patients eligible for standard dose rivaroxaban (4500 on standard dose and 4500 on low dose) found no significant association of dose to risk of ischemic stroke (Table 7).

\section{Major bleeding}

The absolute event rates and event rates/year for bleeding complications in each dosing category are presented in Table 7. Among patients on dabigatran who met criteria for standard dose, use of low dose was associated with significantly higher risk of any major bleeding $(\mathrm{HR}=1.44 ; 95 \%$ CI $1.14-1.8, P=0.002$, Table 7$)$, and higher risk of GI bleeding (HR 1.48, 95\% CI 1,08-2, $P=$ $0.016)$ but not intracranial bleeding compared with patients on standard doses of dabigatran. A similar pattern of increased major bleeding risk (HR 1.34, 95\% CI 1.111.6, $P=0.002)$ was identified among patients on rivaroxaban who met criteria for standard dose but received low dose, along with a trend towards increased risk of GI bleeding (HR 1.26, 95\% CI 0.99-1.6, $P=0.06$ ) but not intracranial bleeding. 
Table 1 Characteristics of patients taking standard $(150 \mathrm{mg})$ or reduced $(75 \mathrm{mg})$ dose dabigatran

\begin{tabular}{|c|c|c|c|}
\hline & $\begin{array}{l}\text { Reduced dose (75 mg twice } \\
\text { daily) }\end{array}$ & $\begin{array}{l}\text { Standard Dose (150 mg twice } \\
\text { daily) }\end{array}$ & $\begin{array}{l}P- \\
\text { value }\end{array}$ \\
\hline Total number of patients & 1455 & 6580 & \\
\hline Age Category, years & & & $<0.001$ \\
\hline $65-69$ & $7.1 \%$ & $18.0 \%$ & \\
\hline 70 to 74 & $13.7 \%$ & $26.8 \%$ & \\
\hline 75 to 79 & $18.8 \%$ & $25.4 \%$ & \\
\hline 80 to 84 & $26.0 \%$ & $18.1 \%$ & \\
\hline 85 to 89 & $16.8 \%$ & $7.1 \%$ & \\
\hline 90 or over & $17.5 \%$ & $4.7 \%$ & \\
\hline Sex & & & $<0.001$ \\
\hline Female & $56.3 \%$ & $47.9 \%$ & \\
\hline Male & $43.7 \%$ & $52.1 \%$ & \\
\hline Race Category & & & $<0.001$ \\
\hline White & $84.1 \%$ & $87.5 \%$ & \\
\hline Black & $10.2 \%$ & $6.9 \%$ & \\
\hline Hispanic & $2.5 \%$ & $1.7 \%$ & \\
\hline Asian & $1.4 \%$ & $0.6 \%$ & \\
\hline Other & $1.9 \%$ & $3.2 \%$ & \\
\hline Smoking & $14.0 \%$ & $16.7 \%$ & 0.01 \\
\hline Alcohol use & $2.0 \%$ & $2.3 \%$ & $\begin{array}{l}< \\
0.001\end{array}$ \\
\hline $\begin{array}{l}\text { Weight Category (based on ICD-9 and ICD-10 codes for BMI } \\
\text { Category) }\end{array}$ & & & $<0.001$ \\
\hline Under-Weight & $1.2 \%$ & $0.6 \%$ & \\
\hline Healthy or Overweight & $10.1 \%$ & $8.0 \%$ & \\
\hline Obese or Severe Obese & $20.1 \%$ & $23.9 \%$ & \\
\hline Not available & $68.5 \%$ & $67.5 \%$ & \\
\hline \multicolumn{4}{|l|}{ Comorbid Conditions } \\
\hline Prior Stroke & $26.8 \%$ & $24.4 \%$ & 0.06 \\
\hline Prior major bleeding from Diagnosis & $26.7 \%$ & $24.5 \%$ & 0.07 \\
\hline Gastrointestinal bleeding & 0 & $13.0 \%$ & 0.24 \\
\hline Cerebral bleeding & 0 & $0.8 \%$ & 0.7 \\
\hline Diabetes & $50.7 \%$ & $48.9 \%$ & 0.2 \\
\hline Prior AMI & $8.0 \%$ & $5.2 \%$ & $\begin{array}{l}< \\
0.001\end{array}$ \\
\hline Liver Disease & $3.4 \%$ & $2.6 \%$ & 0.13 \\
\hline Heart Failure & $48.6 \%$ & $31.3 \%$ & $\begin{array}{l}< \\
0.001\end{array}$ \\
\hline Hypertension & $96.0 \%$ & $93.4 \%$ & $\begin{array}{l}< \\
0.001\end{array}$ \\
\hline Ischemic cardiomyopathy & $55.5 \%$ & $48.0 \%$ & $\begin{array}{l}< \\
0.001\end{array}$ \\
\hline Pulmonary & $16.6 \%$ & $11.4 \%$ & $\begin{array}{l}< \\
0.001\end{array}$ \\
\hline COPD & $36.4 \%$ & $31.8 \%$ & $<001$ \\
\hline Transfusion from Procedure & $3.8 \%$ & $2.7 \%$ & 0.02 \\
\hline
\end{tabular}


Table 1 Characteristics of patients taking standard $(150 \mathrm{mg})$ or reduced $(75 \mathrm{mg})$ dose dabigatran (Continued)

\begin{tabular}{|c|c|c|c|}
\hline & $\begin{array}{l}\text { Reduced dose (75 mg twice } \\
\text { daily) }\end{array}$ & $\begin{array}{l}\text { Standard Dose (150 mg twice } \\
\text { daily) }\end{array}$ & $\begin{array}{l}P- \\
\text { value }\end{array}$ \\
\hline Revascularization & $17.3 \%$ & $15.0 \%$ & 0.03 \\
\hline Implantable Devices & $16.9 \%$ & $13.8 \%$ & 0.002 \\
\hline Valve Disease & $44.7 \%$ & $40.3 \%$ & 0.002 \\
\hline Renal Disease (ICD-9 and ICD-10 codes) & & & $\begin{array}{l}< \\
0.001\end{array}$ \\
\hline None or Mild (Stage I, II) & $53.4 \%$ & $81.7 \%$ & \\
\hline Moderate (Stage III) & $36.0 \%$ & $16.5 \%$ & \\
\hline Severe (Stage IV, V) & $10.6 \%$ & $1.7 \%$ & \\
\hline \multicolumn{4}{|l|}{ Concurrent Drugs (+/- 90 days of initiating DOAC) } \\
\hline SSRI/SNRI & $33.5 \%$ & $31.0 \%$ & 0.05 \\
\hline Strong and moderate P-GP inhibitors & $21.4 \%$ & $22.7 \%$ & 0.28 \\
\hline P-GP inducers & $16.4 \%$ & $18.8 \%$ & 0.03 \\
\hline Strong P-GP and CYP3A4 dual inhibitors & $22.7 \%$ & $22.5 \%$ & 0.8 \\
\hline P-GP and CYP3A4 inducers & $16.4 \%$ & $18.8 \%$ & 0.03 \\
\hline ACE inhibitors & $70.1 \%$ & $68.0 \%$ & 0.12 \\
\hline Warfarin & $20.8 \%$ & $25.0 \%$ & $\begin{array}{l}< \\
0.001\end{array}$ \\
\hline Angiotensin receptor blockers & $41.0 \%$ & $39.3 \%$ & 0.2 \\
\hline Beta blockers & $92.1 \%$ & $89.1 \%$ & $\begin{array}{l}< \\
0.001\end{array}$ \\
\hline Calcium channel blockers & $66.8 \%$ & $63.8 \%$ & 0.03 \\
\hline Digoxin & $31.1 \%$ & $29.4 \%$ & 0.2 \\
\hline Proton pump inhibitors & $60.5 \%$ & $56.4 \%$ & 0.004 \\
\hline NSAIDs & $49.6 \%$ & $51.2 \%$ & 0.29 \\
\hline Antiplatelets & $32.0 \%$ & $26.5 \%$ & $\begin{array}{l}< \\
0.001\end{array}$ \\
\hline Insulin & $17.0 \%$ & $12.9 \%$ & $\begin{array}{l}< \\
0.001\end{array}$ \\
\hline Statins & $79.6 \%$ & $79.4 \%$ & 0.86 \\
\hline Antiarrhythmics & $47.3 \%$ & $48.0 \%$ & 0.6 \\
\hline Estimated Glomerular Filtration rate $(\mathrm{ml} / \mathrm{min} / 1.73 \mathrm{~m} 2)$ & & & $<0.001$ \\
\hline$<30$ & $12.3 \%$ & $2.5 \%$ & \\
\hline $30-60$ & $62.2 \%$ & $45.8 \%$ & \\
\hline $60-90$ & $23.8 \%$ & $46.0 \%$ & \\
\hline$>=90$ & $1.8 \%$ & $5.7 \%$ & \\
\hline CCI(Charlson Comorbodity Index), mean (standard deviation) & $5.52(3.75)$ & $4.17(3.47)$ & $\begin{array}{l}< \\
0.001\end{array}$ \\
\hline
\end{tabular}

Abbreviations: ACE Angiotensin converting enzyme, AMI Acute myocardial infarction, COPD Chronic obstructive pulmonary disease, ICD International classification of diseases, NSAIDS Non-steroidal antiinflammatory drugs, P-GP P-glycoprotein, CYP Cytochrome

In patients who met criteria for low dose dabigatran, there was lower risk of major bleeding $(\mathrm{HR}=0.59 ; 95 \%$ CI $0.43-0.8, P<0.001$, Table 7) and intracranial bleeding ( $\mathrm{HR}=0.33 ; 95 \%$ CI $0.12-0.9, P=0.03$, Table 7) but not GI bleeding in patients who received standard compared to low dose dabigatran. Among patients who met criteria for low dose rivaroxaban, there was lower risk of GI bleeding (HR $=0.79$; 95\% CI 0.64-0.98, $P=0.03$, Table 7) without differences in the risk of major, and intracranial bleeding. After controlling for patient characteristics in propensity-matched samples, we did not find any association of off-label use of low dose or standard dose and the risk of any bleeding events for either dabigatran or rivaroxaban. 
Table 2 Characteristics of patients taking standard $(20 \mathrm{mg})$ or reduced $(15 \mathrm{mg})$ dose rivaroxaban

\begin{tabular}{|c|c|c|c|}
\hline & $\begin{array}{l}\text { Reduced Dose } \\
\text { (15 mg daily) }\end{array}$ & $\begin{array}{l}\text { Standard Dose } \\
\text { (20 mg daily) }\end{array}$ & $P$-value \\
\hline Total number of patients & 6467 & 13,245 & \\
\hline Age Category, years & & & $<0.001$ \\
\hline $65-69$ & $7.9 \%$ & $18.2 \%$ & \\
\hline 70 to 74 & $14.8 \%$ & $29.0 \%$ & \\
\hline 75 to 79 & $20.7 \%$ & $25.6 \%$ & \\
\hline 80 to 84 & $25.4 \%$ & $17.0 \%$ & \\
\hline 85 to 89 & $19.8 \%$ & $7.8 \%$ & \\
\hline 90 or over & $11.4 \%$ & $2.4 \%$ & \\
\hline Sex & & & $<0.001$ \\
\hline Female & $57.1 \%$ & $46.2 \%$ & \\
\hline Male & $42.9 \%$ & $53.8 \%$ & \\
\hline Race Category & & & $<0.001$ \\
\hline White & $85.2 \%$ & $85.8 \%$ & \\
\hline Black & $9.0 \%$ & $8.0 \%$ & \\
\hline Hispanic & $2.2 \%$ & $1.6 \%$ & \\
\hline Asian & $0.8 \%$ & $0.8 \%$ & \\
\hline Other & $2.8 \%$ & $3.8 \%$ & \\
\hline Smoking & $20.2 \%$ & $22.8 \%$ & $<0.001$ \\
\hline Alcohol & $2.3 \%$ & $3.2 \%$ & $<0.001$ \\
\hline Weight Category (based on ICD-9 and ICD-10 codes for BMI Category) & & & $<0.001$ \\
\hline Under-Weight & $2.3 \%$ & $1.3 \%$ & \\
\hline Healthy or Overweight & $19.1 \%$ & $14.3 \%$ & \\
\hline Obese or Severe Obese & $26.2 \%$ & $31.2 \%$ & \\
\hline Not available & $52.4 \%$ & $53.2 \%$ & \\
\hline \multicolumn{4}{|l|}{ Comorbid Conditions } \\
\hline Prior Stroke & $28.2 \%$ & $22.4 \%$ & $<0.001$ \\
\hline Prior major bleeding from Diagnosis & $26.6 \%$ & $23.9 \%$ & $<0.001$ \\
\hline Gastrointestinal bleeding & $15.2 \%$ & $13.5 \%$ & 0.002 \\
\hline Cerebral bleeding & $1.3 \%$ & $0.8 \%$ & $<0.001$ \\
\hline Diabetes & $51.0 \%$ & $47.7 \%$ & $<0.001$ \\
\hline Prior AMI & $9.0 \%$ & $5.7 \%$ & $<0.001$ \\
\hline Liver Disease & $2.9 \%$ & $3.1 \%$ & 0.35 \\
\hline Heart Failure & $35.6 \%$ & $24.3 \%$ & $<0.001$ \\
\hline Hypertension & $94.6 \%$ & $92.1 \%$ & $<0.001$ \\
\hline Ischemic cardiomyopathy & $47.7 \%$ & $40.2 \%$ & $<0.001$ \\
\hline Pulmonary & $15.9 \%$ & $11.1 \%$ & $<0.001$ \\
\hline COPD & $36.3 \%$ & $31.4 \%$ & $<0.001$ \\
\hline Transfusion from Procedure & $5.0 \%$ & $2.3 \%$ & $<0.001$ \\
\hline Revascularization & $16.5 \%$ & $13.7 \%$ & $<0.001$ \\
\hline Implantable Devices & $16.7 \%$ & $12.5 \%$ & $<0.001$ \\
\hline Valve Disease & $40.6 \%$ & $36.4 \%$ & 0.002 \\
\hline Renal Disease (ICD-9 and ICD-10 codes) & & & $<0.001$ \\
\hline None or Mild (Stage I, II) & $54.8 \%$ & $83.9 \%$ & \\
\hline
\end{tabular}


Table 2 Characteristics of patients taking standard $(20 \mathrm{mg})$ or reduced $(15 \mathrm{mg})$ dose rivaroxaban (Continued)

\begin{tabular}{|c|c|c|c|}
\hline & $\begin{array}{l}\text { Reduced Dose } \\
\text { (15 mg daily) }\end{array}$ & $\begin{array}{l}\text { Standard Dose } \\
\text { (20 mg daily) }\end{array}$ & $P$-value \\
\hline Moderate (Stage III) & $37.5 \%$ & $14.5 \%$ & \\
\hline Severe (Stage IV, V) & $7.7 \%$ & $1.5 \%$ & \\
\hline \multicolumn{4}{|l|}{ Concurrent Drugs (+/- 90 days of initiating DOAC) } \\
\hline SSRI/SNRI & $33.7 \%$ & $29.1 \%$ & $<0.001$ \\
\hline Strong and moderate P-GP inhibitors & $18.3 \%$ & $18.5 \%$ & 0.63 \\
\hline P-GP inducers & $18.5 \%$ & $16.5 \%$ & $<0.001$ \\
\hline Strong P-GP and CYP3A4 dual inhibitors & $22.7 \%$ & $20.8 \%$ & 0.002 \\
\hline P-GP and CYP3A4 inducers & $18.5 \%$ & $16.5 \%$ & $<0.001$ \\
\hline ACE inhibitors & $67.4 \%$ & $64.3 \%$ & $<0.001$ \\
\hline Warfarin & $15.0 \%$ & $16.1 \%$ & 0.045 \\
\hline Angiotensin receptor blockers & $40.0 \%$ & $36.7 \%$ & $<0.001$ \\
\hline Beta blockers & $88.1 \%$ & $86.5 \%$ & 0.015 \\
\hline Calcium channel blockers & $64.5 \%$ & $59.4 \%$ & $<0.001$ \\
\hline Digoxin & $23.1 \%$ & $20.8 \%$ & $<0.001$ \\
\hline Proton pump inhibitors & $58.3 \%$ & $52.3 \%$ & $<0.001$ \\
\hline NSAIDs & $52.9 \%$ & $50.1 \%$ & $<0.001$ \\
\hline Antiplatelets & $30.2 \%$ & $24.3 \%$ & $<0.001$ \\
\hline Insulin & $14.3 \%$ & $10.8 \%$ & $<0.001$ \\
\hline Statins & $79.3 \%$ & $77.6 \%$ & 0.006 \\
\hline Antiarrhythmics & $40.4 \%$ & $43.1 \%$ & $<0.001$ \\
\hline Estimated Glomerular Filtration rate $(\mathrm{ml} / \mathrm{min} / 1.73 \mathrm{~m} 2)$ & & & $<0.001$ \\
\hline$<30$ & $7.5 \%$ & $1.3 \%$ & \\
\hline $30-60$ & $61.1 \%$ & $35.2 \%$ & \\
\hline $60-90$ & $28.8 \%$ & $56.7 \%$ & \\
\hline$>=90$ & $2.6 \%$ & $6.8 \%$ & \\
\hline CCI(Charlson Comorbodity Index), mean (standard deviation) & $5.05(3.65)$ & $3.71(3.34)$ & $<0.001$ \\
\hline
\end{tabular}

Abbreviations: ACE Angiotensin converting enzyme, AMI Acute myocardial infarction, COPD Chronic obstructive pulmonary disease, ICD International classification of diseases, NSAIDS Non-steroidal antiinflammatory drugs, P-GP P-glycoprotein, CYP Cytochrome

\section{Sensitivity analysis}

We performed sensitivity analysis among patients with dose adjustments based on valid GFR measurements only and not based on pharmacologic interactions. The propensity matched analysis showed that standard dose dabigatran is associated with higher risk of stroke among patients eligible for low dose according to GFR (HR 2.6, 95\% CI 1.03-6.7; $p=0.04$ ). The analysis did not suggest any other significant differences in stroke and bleeding risks between off-label and standard dosing of dabigatran and rivaroxaban. The results are presented in Additional file 2: Table S2.

\section{Discussion}

The findings of this retrospective cohort analysis of Medicare beneficiaries with AF treated with dabigatran or rivaroxaban between 2010 and 2016 can be summarized as follows: i) among patients on dabigatran or rivaroxaban who met criteria for low dose, the majority received standard dose; ii) among patients on dabigatran or rivaroxaban who met criteria for standard dose, less than one fourth received the low dose; iii) older age, female sex, black race, bleeding history, and heart failure were associated with receipt of lower than recommended dose for patients receiving dabigatran or rivaroxaban; iv) unadjusted analysis suggested that in patients receiving lower dose than recommended, the risk of any major bleeding was increased, likely reflecting higher baseline bleeding risk, while in patients receiving higher dose than recommended, the risk of bleeding was decreased; v) after risk adjusting using multivariable models or propensity-matching, off-label dosing of dabigatran or rivaroxaban was not associated with increased risk of stroke or bleeding compared to recommended dosing. An increased risk of ischemic stroke with standard dose dabigatran was found among patients eligible for low dose based on eGFR only. 
Table 3 Bivariable associations between low dose dabigatran eligible patients' characteristics on low or standard dose of dabigatran

\begin{tabular}{|c|c|c|c|c|c|}
\hline & $\begin{array}{l}\text { Low dose eligible } \\
\text { that received low } \\
\text { dose Dabigatran } \\
(n=442)\end{array}$ & $\begin{array}{l}\text { Low dose eligible } \\
\text { that received } \\
\text { standard dose } \\
\text { Dabigatran } \\
(n=959)\end{array}$ & $\begin{array}{l}P \\
\text { value }\end{array}$ & $\begin{array}{l}\text { Before } \\
\text { Matching } \\
\text { Standardized } \\
\text { Difference }\end{array}$ & $\begin{array}{l}\text { After } \\
\text { Matching } \\
\text { (N=366 vs 366) } \\
\text { Standardized } \\
\text { Difference }\end{array}$ \\
\hline Year & & & 0.001 & & \\
\hline 2010-2012 & $57.7 \%$ & $69.3 \%$ & & 0.244 & 0.228 \\
\hline 2013 & $16.1 \%$ & $10.3 \%$ & & 0.170 & 0.289 \\
\hline 2014 & $10.9 \%$ & $8.1 \%$ & & 0.093 & 0.047 \\
\hline 2015 & $7.7 \%$ & $6.5 \%$ & & 0.048 & 0 \\
\hline 2016-2017 & $7.7 \%$ & $5.7 \%$ & & 0.078 & 0.011 \\
\hline Region & & & 0.1 & & \\
\hline Midwest & $15.4 \%$ & $12.4 \%$ & & 0.086 & 0.008 \\
\hline Northeast & $0.5 \%$ & $1.4 \%$ & & 0.096 & 0.141 \\
\hline South & $73.3 \%$ & $76.7 \%$ & & 0.080 & 0 \\
\hline West & $10.9 \%$ & $9.5 \%$ & & 0.045 & 0.035 \\
\hline Age Category & & & $\begin{array}{l}< \\
0.001\end{array}$ & & \\
\hline $65-69$ & $7.9 \%$ & $14.0 \%$ & & 0.195 & 0 \\
\hline 70 to 74 & $16.5 \%$ & $24.2 \%$ & & 0.192 & 0 \\
\hline 75 to 79 & $18.6 \%$ & $28.5 \%$ & & 0.235 & 0 \\
\hline 80 to 84 & $25.1 \%$ & $19.3 \%$ & & 0.140 & 0 \\
\hline 85 to 89 & $13.1 \%$ & $7.8 \%$ & & 0.174 & 0 \\
\hline 90 or over & $18.8 \%$ & $6.3 \%$ & & 0.385 & 0 \\
\hline Sex & & & 0.2 & & \\
\hline Female & $57.9 \%$ & $54.3 \%$ & & 0.072 & 0 \\
\hline Male & $42.1 \%$ & $45.7 \%$ & & & \\
\hline \multicolumn{6}{|l|}{ Race Category } \\
\hline White & $84.2 \%$ & $85.0 \%$ & & 0.023 & 0 \\
\hline Black & $10.4 \%$ & $8.9 \%$ & & 0.052 & 0 \\
\hline Hispanic & $2.5 \%$ & $2.4 \%$ & & 0.006 & 0 \\
\hline Other & & & & & 0 \\
\hline Smoking & & & 0.04 & & \\
\hline Yes & $11.5 \%$ & $15.7 \%$ & & 0.123 & 0.055 \\
\hline No & $88.5 \%$ & $84.3 \%$ & & & \\
\hline $\begin{array}{l}\text { Weight Category (based on ICD-9 and ICD-10 codes for BMI } \\
\text { Category) }\end{array}$ & & & 0.02 & & \\
\hline Underweight & $1.6 \%$ & $0.6 \%$ & & 0.092 & 0 \\
\hline Healthy or Overweight & $8.6 \%$ & $6.3 \%$ & & 0.089 & 0.062 \\
\hline Obese or Severe Obese & $20.4 \%$ & $26.3 \%$ & & 0.140 & 0.013 \\
\hline Others & $69.5 \%$ & $66.8 \%$ & & 0.056 & 0.024 \\
\hline \multicolumn{6}{|l|}{ Comorbid Conditions } \\
\hline Prior Stroke & $26.7 \%$ & $27.4 \%$ & 0.8 & 0.016 & 0.042 \\
\hline Prior major bleeding & $29.0 \%$ & $28.2 \%$ & 0.8 & 0.018 & 0.006 \\
\hline Diabetes & $55.0 \%$ & $54.0 \%$ & 0.7 & 0.019 & 0.077 \\
\hline Prior AMI & $10.6 \%$ & $6.2 \%$ & 0.003 & 0.162 & 0.147 \\
\hline Liver Disease & $2.9 \%$ & $3.2 \%$ & 0.8 & 0.017 & 0 \\
\hline
\end{tabular}


Table 3 Bivariable associations between low dose dabigatran eligible patients' characteristics on low or standard dose of dabigatran (Continued)

\begin{tabular}{|c|c|c|c|c|c|}
\hline & $\begin{array}{l}\text { Low dose eligible } \\
\text { that received low } \\
\text { dose Dabigatran } \\
(n=442)\end{array}$ & $\begin{array}{l}\text { Low dose eligible } \\
\text { that received } \\
\text { standard dose } \\
\text { Dabigatran } \\
(n=959)\end{array}$ & $\begin{array}{l}P \\
\text { value }\end{array}$ & $\begin{array}{l}\text { Before } \\
\text { Matching } \\
\text { Standardized } \\
\text { Difference }\end{array}$ & $\begin{array}{l}\text { After } \\
\text { Matching } \\
\text { ( } N=366 \text { vs 366) } \\
\text { Standardized } \\
\text { Difference } \\
\end{array}$ \\
\hline Heart Failure & $56.8 \%$ & $40.7 \%$ & $\begin{array}{l}< \\
0.001\end{array}$ & 0.327 & 0.265 \\
\hline Hypertension & $98.2 \%$ & $96.1 \%$ & 0.04 & 0.124 & 0.082 \\
\hline Ischemic cardiomyopathy & $60.4 \%$ & $57.2 \%$ & 0.3 & 0.064 & 0.089 \\
\hline Pulmonary & $18.8 \%$ & $13.3 \%$ & 0.008 & 0.148 & 0.066 \\
\hline COPD & $37.8 \%$ & $36.2 \%$ & 0.6 & 0.033 & 0.034 \\
\hline Transfusion from Procedure & $5.4 \%$ & $4.3 \%$ & 0.3 & 0.054 & 0.051 \\
\hline Revascularization & $18.6 \%$ & $17.8 \%$ & 0.7 & 0.019 & 0.043 \\
\hline Implantable Devices & $20.1 \%$ & $17.5 \%$ & 0.2 & 0.067 & 0 \\
\hline Valve Disease & $45.9 \%$ & $42.0 \%$ & 0.2 & 0.079 & 0.099 \\
\hline \multicolumn{6}{|c|}{ Concurrent Drugs ( $+/-90$ days of initiating DOAC) } \\
\hline SSRI/SNRI & $32.6 \%$ & $36.5 \%$ & 0.15 & 0.083 & 0.023 \\
\hline Strong and moderate p-gp inhibitors & $53.5 \%$ & $79.9 \%$ & $\begin{array}{l}< \\
0.001\end{array}$ & 0.580 & 0.068 \\
\hline Warfarin & $22.6 \%$ & $31.0 \%$ & 0.001 & 0.189 & 0.153 \\
\hline Strong p-gp and cyp3a4 dual inhibitors & $35.7 \%$ & $47.5 \%$ & $\begin{array}{l}< \\
0.001\end{array}$ & 0.241 & 0.089 \\
\hline ACE inhibitors & $74.0 \%$ & $72.7 \%$ & 0.6 & 0.029 & 0.044 \\
\hline Angiotensin receptor blockers & $46.6 \%$ & $45.9 \%$ & 0.8 & 0.015 & 0.066 \\
\hline Beta blockers & $95.2 \%$ & $92.9 \%$ & 0.09 & 0.099 & 0.165 \\
\hline Calcium channel blockers & $69.7 \%$ & $67.5 \%$ & 0.4 & 0.048 & 0.098 \\
\hline Digoxin & $28.7 \%$ & $33.8 \%$ & 0.06 & 0.109 & 0.083 \\
\hline Proton pump inhibitors & $64.7 \%$ & $63.2 \%$ & 0.6 & 0.032 & 0.119 \\
\hline NSAIDs & $52.7 \%$ & $55.4 \%$ & 0.4 & 0.053 & 0.055 \\
\hline Antiplatelets & $35.3 \%$ & $31.3 \%$ & 0.13 & 0.085 & 0.099 \\
\hline Insulin & $22.9 \%$ & $17.6 \%$ & 0.02 & 0.130 & 0.206 \\
\hline Statins & $80.8 \%$ & $83.3 \%$ & 0.24 & 0.066 & 0.007 \\
\hline \multicolumn{6}{|l|}{ Renal Disease } \\
\hline Moderate (GFR 30-60 ml/min/1.73 m2) & $45.7 \%$ & $74.9 \%$ & $\begin{array}{l}< \\
0.001\end{array}$ & 0.6245 & 0.0439 \\
\hline $\begin{array}{l}\text { Severe } \\
(\mathrm{GFR}<30 \mathrm{ml} / \mathrm{min} / 1.73 \mathrm{~m} 2)\end{array}$ & $54.3 \%$ & $25.1 \%$ & & & \\
\hline
\end{tabular}

Abbreviations: $A C E$ Angiotensin converting enzyme, $A M I$ Acute myocardial infarction, COPD Chronic obstructive pulmonary disease, ICD International classification of diseases, NSAIDS Non-steroidal antiinflammatory drugs, P-GP P-glycoprotein, CYP Cytochrome

The results of our analysis are in accordance with findings of previous studies which demonstrated that a significant part of AF population on DOACs receive an off-label dose $[14,15,19]$. An updated analysis of the ORBITA-AF II registry from 2013 until 2016, including 7925 AF patients treated with DOACs, showed that $84 \%$ received DOACs at standard dose (mainly rivaroxaban and apixaban, only 451 patients on dabigatran), which was consistent with FDA labeling in 96\% of cases [15]. Reduced DOAC dose was prescribed to $16 \%$ of patients, which was consistent with FDA labeling in 43\%. In unadjusted analysis, under-dosing was associated with higher rates of all-cause mortality and major bleeding [15]. Nevertheless, after risk- adjustment, the use of lower-thanrecommended dose resulted in similar thromboembolic and bleeding risk compared to appropriately dosed DOAC use [15]. Our cohort included a larger sample size than ORBITA-AF II and focused on dabigatran and rivaroxaban as opposed to apixaban and rivaroxaban in ORBITA-AF II. Another methodological difference is the use of calculated 
Table 4 Bivariable associations between standard dose dabigatran eligible patients' characteristics on low or standard dose of dabigatran

\begin{tabular}{|c|c|c|c|c|c|}
\hline & $\begin{array}{l}\text { Standard dose eligible that } \\
\text { received low dose Dabigatran } \\
(n=1013)\end{array}$ & $\begin{array}{l}\text { Standard dose eligible } \\
\text { that received standard } \\
\text { dose Dabigatran } \\
(n=5621)\end{array}$ & $\begin{array}{l}P \\
\text { value }\end{array}$ & $\begin{array}{l}\text { Before } \\
\text { Matching } \\
\text { Standardized } \\
\text { Difference }\end{array}$ & $\begin{array}{l}\text { After Matching } \\
(N=1001 \text { vs } \\
1001) \\
\text { Standardized } \\
\text { Difference }\end{array}$ \\
\hline Year & & & $\begin{array}{l}< \\
0.001\end{array}$ & & \\
\hline 2010-12 & $50.0 \%$ & $55.7 \%$ & & 0.116 & 0.198 \\
\hline 2013 & $18.6 \%$ & $13.6 \%$ & & 0.137 & 0.174 \\
\hline 2014 & $11.6 \%$ & $11.4 \%$ & & 0.008 & 0.032 \\
\hline 2015 & $10.9 \%$ & $10.1 \%$ & & 0.026 & 0.047 \\
\hline 2016-17 & $9.0 \%$ & $9.3 \%$ & & 0.009 & 0.040 \\
\hline Region & & & 0.8 & & \\
\hline Midwest & $12.3 \%$ & $12.9 \%$ & & 0.017 & 0.024 \\
\hline Northeast & $1.7 \%$ & $1.5 \%$ & & 0.018 & 0.016 \\
\hline South & $73.7 \%$ & $74.3 \%$ & & 0.012 & 0.009 \\
\hline West & $12.2 \%$ & $11.4 \%$ & & 0.027 & 0.006 \\
\hline Age Category & & & $\begin{array}{l}< \\
0.001\end{array}$ & & \\
\hline $65-69$ & $6.7 \%$ & $18.7 \%$ & & 0.365 & 0 \\
\hline 70 to 74 & $12.5 \%$ & $27.3 \%$ & & 0.3755 & 0 \\
\hline 75 to 79 & $19.0 \%$ & $24.9 \%$ & & 0.143 & 0 \\
\hline 80 to 84 & $26.4 \%$ & $17.9 \%$ & & 0.206 & 0 \\
\hline 85 to 89 & $18.5 \%$ & $6.9 \%$ & & 0.351 & 0 \\
\hline 90 or over & $17.0 \%$ & $4.4 \%$ & & 0.415 & 0 \\
\hline Sex & & & $\begin{array}{l}< \\
0.001\end{array}$ & & \\
\hline Female & $55.6 \%$ & $46.8 \%$ & & 0.176 & 0 \\
\hline Male & $44.4 \%$ & $53.2 \%$ & & & \\
\hline Race Category & & & $\begin{array}{l}< \\
0.001\end{array}$ & & \\
\hline White & $84.0 \%$ & $87.9 \%$ & & 0.113 & 0 \\
\hline Black & $10.1 \%$ & $6.6 \%$ & & 0.126 & 0 \\
\hline Hispanic & $2.5 \%$ & $1.6 \%$ & & 0.059 & 0 \\
\hline Asian & $1.2 \%$ & $0.6 \%$ & & 0.066 & 0 \\
\hline Other & $2.3 \%$ & $3.3 \%$ & & 0.060 & 0 \\
\hline Smoker & $15.0 \%$ & $16.8 \%$ & 0.15 & 0.049 & 0.037 \\
\hline $\begin{array}{l}\text { Weight Category (based on ICD-9/ ICD-10 codes for } \\
\text { BMI) }\end{array}$ & & & 0.003 & & \\
\hline Under Weight & $1.1 \%$ & $0.6 \%$ & & 0.057 & 0.031 \\
\hline Healthy or Overweight & $10.8 \%$ & $8.3 \%$ & & 0.082 & 0.013 \\
\hline Obese or Severe Obese & $20.0 \%$ & $23.4 \%$ & & 0.084 & 0.054 \\
\hline Others & $68.1 \%$ & $67.6 \%$ & & 0.010 & 0.061 \\
\hline \multicolumn{6}{|l|}{ Comorbid Conditions } \\
\hline Prior Stroke & $26.9 \%$ & $23.9 \%$ & 0.04 & 0.067 & 0.011 \\
\hline Prior Major Bleeding & $25.8 \%$ & $23.9 \%$ & 0.2 & 0.044 & 0.042 \\
\hline Diabetes & $48.9 \%$ & $48.0 \%$ & 0.6 & 0.018 & 0.066 \\
\hline
\end{tabular}


Table 4 Bivariable associations between standard dose dabigatran eligible patients' characteristics on low or standard dose of dabigatran (Continued)

\begin{tabular}{|c|c|c|c|c|c|}
\hline & $\begin{array}{l}\text { Standard dose eligible that } \\
\text { received low dose Dabigatran } \\
(n=1013)\end{array}$ & $\begin{array}{l}\text { Standard dose eligible } \\
\text { that received standard } \\
\text { dose Dabigatran } \\
(n=5621)\end{array}$ & $\begin{array}{l}P \\
\text { value }\end{array}$ & $\begin{array}{l}\text { Before } \\
\text { Matching } \\
\text { Standardized } \\
\text { Difference }\end{array}$ & $\begin{array}{l}\text { After Matching } \\
(N=1001 \text { vs } \\
1001) \\
\text { Standardized } \\
\text { Difference }\end{array}$ \\
\hline Prior AMI & $6.9 \%$ & $5.0 \%$ & 0.01 & 0.082 & 0.103 \\
\hline Liver Disease & $3.6 \%$ & $2.5 \%$ & 0.07 & 0.059 & 0.126 \\
\hline Heart Failure & $45.0 \%$ & $29.7 \%$ & $\begin{array}{l}< \\
0.001\end{array}$ & 0.320 & 0.274 \\
\hline Hypertension & $95.1 \%$ & $93.0 \%$ & 0.01 & 0.089 & 0.088 \\
\hline Ischemic Cardiomyopathy & $53.4 \%$ & $46.4 \%$ & $\begin{array}{l}< \\
0.001\end{array}$ & 0.140 & 0.110 \\
\hline Pulmonary Circulatory Disease & $15.6 \%$ & $11.0 \%$ & $\begin{array}{l}< \\
0.001\end{array}$ & 0.135 & 0.083 \\
\hline COPD & $35.7 \%$ & $31.0 \%$ & 0.003 & 0.1 & 0.115 \\
\hline Blood Transfusion & $3.2 \%$ & $2.4 \%$ & 0.18 & 0.044 & 0 \\
\hline Revascularization & $16.8 \%$ & $14.5 \%$ & 0.05 & 0.063 & 0.075 \\
\hline Implantable cardiac device & $15.5 \%$ & $13.1 \%$ & 0.04 & 0.068 & 0.011 \\
\hline Valve Disease & $44.1 \%$ & $40.0 \%$ & 0.015 & 0.083 & 0.024 \\
\hline \multicolumn{6}{|c|}{ Concurrent Drugs (+/- 90 days of initiating DOAC) } \\
\hline SSRI/SNRI & $34.0 \%$ & $30.0 \%$ & 0.01 & 0.085 & 0.126 \\
\hline Strong and moderate p-gp inhibitors & $7.3 \%$ & $12.9 \%$ & $\begin{array}{l}< \\
0.001\end{array}$ & 0.188 & 0.135 \\
\hline Warfarin & $19.9 \%$ & $24.0 \%$ & 0.004 & 0.099 & 0.237 \\
\hline Strong p-gp and cyp3a4 dual inhibitors & $17.0 \%$ & $18.2 \%$ & 0.3 & 0.0325 & 0.032 \\
\hline ACE inhibitors & $68.4 \%$ & $67.2 \%$ & 0.45 & 0.0257 & 0.081 \\
\hline Angiotensin receptor blockers & $38.6 \%$ & $38.1 \%$ & 0.8 & 0.0094 & 0.010 \\
\hline Beta blockers & $90.7 \%$ & $88.4 \%$ & 0.03 & 0.0759 & 0.108 \\
\hline Calcium channel blockers & $65.5 \%$ & $63.2 \%$ & 0.15 & 0.0488 & 0.040 \\
\hline Digoxin & $32.1 \%$ & $28.7 \%$ & 0.03 & 0.0741 & 0.002 \\
\hline Proton pump inhibitors & $58.7 \%$ & $55.2 \%$ & 0.04 & 0.0707 & 0.008 \\
\hline NSAIDs & $48.3 \%$ & $50.4 \%$ & 0.2 & 0.0433 & 0.04 \\
\hline Antiplatelets & $30.5 \%$ & $25.7 \%$ & 0.0015 & 0.1064 & 0.093 \\
\hline Insulin & $14.5 \%$ & $12.1 \%$ & 0.03 & 0.072 & 0.137 \\
\hline Statins & $79.1 \%$ & $78.7 \%$ & 0.8 & 0.009 & 0.079 \\
\hline Renal Disease & & & $\begin{array}{l}< \\
0.001\end{array}$ & & \\
\hline None or Mild & $33.6 \%$ & $57.2 \%$ & & 0.489 & 0.255 \\
\hline Moderate (GFR 30-60 ml/min/1.73 m2) & $66.4 \%$ & $42.8 \%$ & & & \\
\hline Severe (GFR < $30 \mathrm{ml} / \mathrm{min} / 1.73 \mathrm{~m} 2)$ & $0.0 \%$ & $0.0 \%$ & & & \\
\hline
\end{tabular}

Abbreviations: ACE Angiotensin converting enzyme, AMI Acute myocardial infarction, COPD Chronic obstructive pulmonary disease, ICD International classification of diseases, NSAIDS Non-steroidal antiinflammatory drugs, P-GP P-glycoprotein, CYP Cytochrome

creatinine clearance with the Cockcroft-Gault formula instead of the MDRD or the CKD-EPI equations for eGFR calculation that we applied. Although in clinical practice, eGFR by MDRD or CKD-EPI is more commonly used than calculated creatinine clearance, discordances in dabigatran and rivaroxaban doses may occur in up to $30 \%$ of elderly patients with creatinine clearance $<60 \mathrm{ml} / \mathrm{min}$ [ 19]. Despite these methodological differences both our analysis and the previous report from the ORBITA-AF II registry suggest that among patients on dabigatran or rivaroxaban who met criteria for low dose, the majority received standard dose. Moreover, unadjusted analysis suggested that in patients 
Table 5 Bivariable associations between low dose rivaroxaban eligible patients' characteristics on low or standard dose of rivaroxaban

\begin{tabular}{|c|c|c|c|c|c|}
\hline & $\begin{array}{l}\text { Low dose eligible that } \\
\text { received low dose } \\
\text { Rivaroxaban } \\
(n=3916)\end{array}$ & $\begin{array}{l}\text { Low dose eligible that } \\
\text { received standard dose } \\
\text { Rivaroxaban }(n=3904)\end{array}$ & $P$ value & $\begin{array}{l}\text { Before } \\
\text { Matching } \\
\text { Standardized } \\
\text { Difference }\end{array}$ & $\begin{array}{l}\text { After } \\
\text { Matching } \\
(N=2703 \\
\text { vs 2703) } \\
\text { Standardized } \\
\text { Difference }\end{array}$ \\
\hline Year & & & 0.2 & & \\
\hline 2010-2012 & $7.2 \%$ & $6.7 \%$ & & 0.021 & 0.028 \\
\hline 2013 & $18.3 \%$ & $17.6 \%$ & & 0.017 & 0.0308 \\
\hline 2014 & $23.0 \%$ & $23.0 \%$ & & 0.002 & 0.016 \\
\hline 2015 & $22.9 \%$ & $21.8 \%$ & & 0.024 & 0.003 \\
\hline 2016-2017 & $28.6 \%$ & $30.9 \%$ & & 0.050 & 0.058 \\
\hline Region & & & 0.04 & & \\
\hline Midwest & $15.2 \%$ & $13.0 \%$ & & 0.064 & 0.084 \\
\hline Northeast & $1.2 \%$ & $1.1 \%$ & & 0.009 & 0.016 \\
\hline South & $73.0 \%$ & $75.0 \%$ & & 0.044 & 0.028 \\
\hline West & $10.5 \%$ & $10.9 \%$ & & 0.013 & 0.061 \\
\hline Age Category & & & $<0.001$ & & \\
\hline $65-69$ & $7.5 \%$ & $14.5 \%$ & & 0.225 & 0 \\
\hline 70 to 74 & $14.0 \%$ & $26.0 \%$ & & 0.302 & 0 \\
\hline 75 to 79 & $20.1 \%$ & $25.8 \%$ & & 0.135 & 0 \\
\hline 80 to 84 & $25.5 \%$ & $19.7 \%$ & & 0.139 & 0 \\
\hline 85 to 89 & $20.5 \%$ & $10.1 \%$ & & 0.291 & 0 \\
\hline 90 or over & $12.4 \%$ & $3.9 \%$ & & 0.311 & 0 \\
\hline Sex & & & 0.0008 & & \\
\hline Female & $56.0 \%$ & $52.3 \%$ & & 0.076 & 0 \\
\hline Male & $44.0 \%$ & $47.7 \%$ & & & \\
\hline Race Category & & & 0.7 & & \\
\hline White & $84.5 \%$ & $83.4 \%$ & & 0.031 & 0 \\
\hline Black & $10.2 \%$ & $10.7 \%$ & & 0.017 & 0 \\
\hline Hispanic & $2.0 \%$ & $2.2 \%$ & & 0.011 & 0 \\
\hline Asian & $0.7 \%$ & $0.8 \%$ & & 0.015 & 0 \\
\hline Other & $2.7 \%$ & $3.0 \%$ & & 0.019 & 0 \\
\hline Smoker & $19.6 \%$ & $22.5 \%$ & 0.001 & 0.073 & 0.033 \\
\hline Weight Category (ICD9/ICD-10 codes for BMI) & & & $<0.0001$ & & \\
\hline Under Weight & $1.9 \%$ & $1.2 \%$ & & 0.053 & 0.025 \\
\hline Healthy or Overweight & $18.4 \%$ & $16.1 \%$ & & 0.062 & 0.008 \\
\hline Obese or Severe Obese & $28.9 \%$ & $35.4 \%$ & & 0.139 & 0.079 \\
\hline Others & $50.8 \%$ & $47.3 \%$ & & 0.070 & 0.062 \\
\hline \multicolumn{6}{|l|}{ Comorbid Conditions } \\
\hline Prior Stroke & $29.1 \%$ & $25.1 \%$ & $<0.001$ & 0.089 & 0.051 \\
\hline Prior major bleeding & $27.6 \%$ & $28.0 \%$ & 0.6 & 0.011 & 0.026 \\
\hline Diabetes & $55.9 \%$ & $57.2 \%$ & 0.2 & 0.027 & 0.021 \\
\hline Prior AMI & $10.4 \%$ & $6.7 \%$ & $<0.001$ & 0.132 & 0.119 \\
\hline Liver Disease & $3.2 \%$ & $3.5 \%$ & 0.4 & 0.019 & 0.017 \\
\hline Heart Failure & $41.2 \%$ & $31.6 \%$ & $<0.001$ & 0.201 & 0.151 \\
\hline
\end{tabular}


Table 5 Bivariable associations between low dose rivaroxaban eligible patients' characteristics on low or standard dose of rivaroxaban (Continued)

\begin{tabular}{|c|c|c|c|c|c|}
\hline & $\begin{array}{l}\text { Low dose eligible that } \\
\text { received low dose } \\
\text { Rivaroxaban } \\
(n=3916)\end{array}$ & $\begin{array}{l}\text { Low dose eligible that } \\
\text { received standard dose } \\
\text { Rivaroxaban }(n=3904)\end{array}$ & $P$ value & $\begin{array}{l}\text { Before } \\
\text { Matching } \\
\text { Standardized } \\
\text { Difference }\end{array}$ & $\begin{array}{l}\text { After } \\
\text { Matching } \\
(N=2703 \\
\text { vs 2703) } \\
\text { Standardized } \\
\text { Difference } \\
\end{array}$ \\
\hline Hypertension & $96.8 \%$ & $95.3 \%$ & $<0.001$ & 0.076 & 0.030 \\
\hline Cardiomyopathy & $50.4 \%$ & $44.6 \%$ & $<0.001$ & 0.115 & 0.100 \\
\hline Pulmonary Circulatory Dis & $17.2 \%$ & $14.2 \%$ & $<0.001$ & 0.082 & 0.021 \\
\hline COPD & $38.5 \%$ & $37.0 \%$ & 0.17 & 0.031 & 0.054 \\
\hline Prior Blood Transfusion & $5.8 \%$ & $3.5 \%$ & $<0.001$ & 0.107 & 0.083 \\
\hline Revascularization & $17.7 \%$ & $15.4 \%$ & 0.007 & 0.061 & 0.079 \\
\hline Implantable Cardiac Device & $18.3 \%$ & $14.8 \%$ & $<0.001$ & 0.094 & 0.078 \\
\hline Valve Disease & $42.2 \%$ & $38.1 \%$ & $<0.001$ & 0.084 & 0.064 \\
\hline \multicolumn{6}{|c|}{ Concurrent Drugs (+/- 90 days of initiating DOAC) } \\
\hline SSRI/SNRI & $34.0 \%$ & $33.3 \%$ & 0.5 & 0.014 & 0.034 \\
\hline Strong and moderate $\mathrm{p}$-gp inhibitors & $19.9 \%$ & $24.5 \%$ & $<0.001$ & 0.110 & 0.012 \\
\hline Warfarin & $16.1 \%$ & $19.6 \%$ & $<0.001$ & 0.092 & 0.124 \\
\hline Pgp inducers & $17.9 \%$ & $18.0 \%$ & 0.9 & 0.003 & 0.018 \\
\hline Strong p-gp and cyp3a4 dual inhibitors & $26.1 \%$ & $35.1 \%$ & $<0.001$ & 0.194 & 0.009 \\
\hline P-gp and cyp3a4 inducers & $17.9 \%$ & $18.1 \%$ & 0.9 & 0.003 & 0.017 \\
\hline Ace inhibitors & $70.6 \%$ & $69.6 \%$ & 0.34 & 0.022 & 0.025 \\
\hline Angiotensin receptor blockers & $43.9 \%$ & $43.8 \%$ & 0.86 & 0.004 & 0.004 \\
\hline Beta blockers & $89.7 \%$ & $89.0 \%$ & 0.32 & 0.023 & 0.019 \\
\hline Calcium channel blockers & $67.6 \%$ & $64.4 \%$ & $=0.003$ & 0.067 & 0.026 \\
\hline Digoxin & $23.0 \%$ & $23.3 \%$ & 0.7 & 0.008 & 0.003 \\
\hline Proton pump inhibitors & $59.6 \%$ & $59.2 \%$ & 0.7 & 0.008 & 0.026 \\
\hline NSAIDS & $52.6 \%$ & $53.3 \%$ & 0.5 & 0.015 & 0.022 \\
\hline Antiplatelets & $31.8 \%$ & $28.4 \%$ & 0.001 & 0.074 & 0.067 \\
\hline Insulin & $18.0 \%$ & $17.1 \%$ & 0.27 & 0.025 & 0.087 \\
\hline Statins & $81.8 \%$ & $81.6 \%$ & 0.79 & 0.006 & 0.024 \\
\hline \multicolumn{6}{|l|}{ Renal Disease } \\
\hline None or mild & $3.6 \%$ & $17.1 \%$ & $<0.0001$ & 0.452 & 0.058 \\
\hline Moderate (GFR 30-60 ml/min/1.73 m2) & $83.3 \%$ & $77.4 \%$ & & 0.149 & 0.039 \\
\hline $\begin{array}{l}\text { Severe } \\
(\text { GFR }<30 \mathrm{ml} / \mathrm{min} / 1.73 \mathrm{~m} 2)\end{array}$ & $13.1 \%$ & $5.5 \%$ & & 0.262 & 0.002 \\
\hline
\end{tabular}

Abbreviations: ACE Angiotensin converting enzyme, $A M I$ Acute myocardial infarction, COPD Chronic obstructive pulmonary disease, ICD International classification of diseases, NSAIDS Non-steroidal antiinflammatory drugs, P-GP P-glycoprotein, CYP Cytochrome

receiving lower dose than recommended, the risk of any major bleeding was increased. In both analyses, propensity matching did not reveal any significant differences in stroke and bleeding.

Yao et al. ${ }^{19}$ previously evaluated potential over- and under- dosing of DOACs based only on renal indication for dose reduction using the data for privately insured and Medicare Advantage enrollees. Like our study, Yao et al. found no significant relationship between risk of stroke or bleeding and dose in dabigatran or rivaroxaban-treated patients with renal indication for dose reduction. However, in aggregate analyses of patients taking dabigatran, rivaroxaban or apixaban with renal indication for low dose, patients had significantly higher bleeding risk if they received standard dose. Consistent with our study, Yao et al. also found no statistically significant relationship between dose reduction and risk of stroke or bleeding in the dabigatran- or rivaroxaban-treated patients who did not have a renal indication for low dose. In contrast to our study, Yao et al. evaluated renal indications for dose 
Table 6 Bivariable associations between standard dose rivaroxaban eligible patients' characteristics on low or standard dose of rivaroxaban

\begin{tabular}{|c|c|c|c|c|c|}
\hline & $\begin{array}{l}\text { Standard dose eligible } \\
\text { that received low dose } \\
\text { Rivaroxaban }(n=2551)\end{array}$ & $\begin{array}{l}\text { Standard dose eligible } \\
\text { that received standard } \\
\text { dose Rivaroxaban } \\
(n=9341)\end{array}$ & $P$ value & $\begin{array}{l}\text { Before Matching } \\
\text { Standardized } \\
\text { Difference }\end{array}$ & $\begin{array}{l}\text { After Matching } \\
(N=2397 \text { vs } \\
2397) \\
\text { Standardized } \\
\text { Difference }\end{array}$ \\
\hline Year & & & 0.06 & & \\
\hline 2010-2012 & $8.2 \%$ & $7.1 \%$ & & 0.0439 & 0.0031 \\
\hline 2013 & $17.9 \%$ & $17.1 \%$ & & 0.0204 & 0.0098 \\
\hline 2014 & $23.8 \%$ & $22.7 \%$ & & 0.0256 & 0.0248 \\
\hline 2015 & $21.4 \%$ & $22.7 \%$ & & 0.0312 & 0.0051 \\
\hline $2016-2017$ & $28.7 \%$ & $30.4 \%$ & & 0.0379 & 0.0284 \\
\hline Region & & & 0.12 & & \\
\hline Midwest & $15.1 \%$ & $14.4 \%$ & & 0.0178 & 0.1713 \\
\hline Northeast & $1.1 \%$ & $1.3 \%$ & & 0.0229 & 0.1515 \\
\hline South & $73.1 \%$ & $72.0 \%$ & & 0.0254 & 0.2038 \\
\hline West & $10.8 \%$ & $12.3 \%$ & & 0.0476 & 0.0186 \\
\hline Age Category & & & $<0.0001$ & & \\
\hline $65-69$ & $8.5 \%$ & $19.7 \%$ & & 0.3266 & 0 \\
\hline 70 to 74 & $15.9 \%$ & $30.3 \%$ & & 0.3453 & 0 \\
\hline 75 to 79 & $21.6 \%$ & $25.5 \%$ & & 0.0916 & 0 \\
\hline 80 to 84 & $25.3 \%$ & $15.9 \%$ & & 0.2337 & 0 \\
\hline 85 to 89 & $18.8 \%$ & $6.9 \%$ & & 0.3622 & 0 \\
\hline 90 or over & $9.8 \%$ & $1.7 \%$ & & 0.3544 & 0 \\
\hline Sex & & & $<0.0001$ & & \\
\hline Female & $58.7 \%$ & $43.7 \%$ & & 0.3044 & 0 \\
\hline Male & $41.3 \%$ & $56.3 \%$ & & & \\
\hline Race Category & & & $<0.0001$ & & \\
\hline White & $86.2 \%$ & $86.8 \%$ & & 0.0164 & 0 \\
\hline Black & $7.3 \%$ & $6.9 \%$ & & 0.0144 & 0 \\
\hline Hispanic & $2.4 \%$ & $1.3 \%$ & & 0.0813 & 0 \\
\hline Asian & $1.1 \%$ & $0.8 \%$ & & 0.0304 & 0 \\
\hline Other & $3.0 \%$ & $4.2 \%$ & & 0.065 & 0 \\
\hline Smoker & $21.1 \%$ & $23.0 \%$ & 0.05 & 0.044 & 0.003 \\
\hline Weight Category (based on ICD-9/ICD-10 codes for BMI) & & & $<0.0001$ & & \\
\hline Under & $2.9 \%$ & $1.4 \%$ & & 0.1038 & 0.0682 \\
\hline Healthy or Overweight & $20.1 \%$ & $13.6 \%$ & & 0.1737 & 0.2328 \\
\hline Obese or Severe Obese & $22.1 \%$ & $29.4 \%$ & & 0.1684 & 0.0561 \\
\hline Others & $55.0 \%$ & $55.6 \%$ & & 0.0126 & 0.2406 \\
\hline \multicolumn{6}{|l|}{ Comorbid Conditions } \\
\hline Prior Stroke & $26.9 \%$ & $21.3 \%$ & $<0.001$ & 0.1295 & 0 \\
\hline Prior major bleeding & $25.2 \%$ & $22.2 \%$ & 0.001 & 0.071 & 0 \\
\hline Diabetes & $43.4 \%$ & $43.8 \%$ & 0.76 & 0.0069 & 0.1063 \\
\hline Prior AMl & $7.0 \%$ & $5.3 \%$ & 0.001 & 0.0701 & 0 \\
\hline Liver Disease & $2.4 \%$ & $2.9 \%$ & 0.14 & 0.0337 & 0.0139 \\
\hline Heart Failure & $27.0 \%$ & $21.3 \%$ & $<0.001$ & 0.1339 & 0 \\
\hline Hypertension & $91.2 \%$ & $90.8 \%$ & 0.57 & 0.0128 & 0.0474 \\
\hline Cardiomyopathy & $43.5 \%$ & $38.3 \%$ & $<0.001$ & 0.1063 & 0.1134 \\
\hline Pulmonary Circulatory dis & $13.9 \%$ & $9.8 \%$ & $<0.001$ & 0.1277 & 0.0414 \\
\hline COPD & $33.0 \%$ & $29.1 \%$ & $<0.001$ & 0.0846 & 0.0972 \\
\hline
\end{tabular}


Table 6 Bivariable associations between standard dose rivaroxaban eligible patients' characteristics on low or standard dose of rivaroxaban (Continued)

\begin{tabular}{|c|c|c|c|c|c|}
\hline & $\begin{array}{l}\text { Standard dose eligible } \\
\text { that received low dose } \\
\text { Rivaroxaban ( } n=2551)\end{array}$ & $\begin{array}{l}\text { Standard dose eligible } \\
\text { that received standard } \\
\text { dose Rivaroxaban } \\
(n=9341)\end{array}$ & $P$ value & $\begin{array}{l}\text { Before Matching } \\
\text { Standardized } \\
\text { Difference }\end{array}$ & $\begin{array}{l}\text { After Matching } \\
(N=2397 \text { vs } \\
2397) \\
\text { Standardized } \\
\text { Difference }\end{array}$ \\
\hline Prior blood transfusion & $3.7 \%$ & $1.8 \%$ & $<0.001$ & 0.1173 & 0.0855 \\
\hline Revascularization & $14.7 \%$ & $13.0 \%$ & 0.02 & 0.0495 & 0.08 \\
\hline Implantable Devices & $14.1 \%$ & $11.6 \%$ & $<0.001$ & 0.0766 & 0.0553 \\
\hline Valve Disease & $38.1 \%$ & $35.7 \%$ & 0.02 & 0.051 & 0.0347 \\
\hline \multicolumn{6}{|c|}{ Concurrent Drugs (+/- 90 days of initiating DOAC) } \\
\hline SSRI/SNRI & $33.3 \%$ & $27.4 \%$ & $<0.001$ & 0.1289 & 0.0682 \\
\hline Strong and moderate p-gp inhibitors & $15.7 \%$ & $16.1 \%$ & 0.67 & 0.0096 & 0 \\
\hline Warfarin & $13.2 \%$ & $14.6 \%$ & 0.08 & 0.0391 & 0.1199 \\
\hline Pgp inducers & $19.5 \%$ & $15.8 \%$ & $<0.001$ & 0.097 & 0 \\
\hline Strong p-gp and cyp3a4 dual inhibitors & $17.3 \%$ & $14.8 \%$ & 0.002 & 0.069 & 0.0232 \\
\hline P-gp and cyp3a4 inducers & $19.5 \%$ & $15.8 \%$ & $<0.001$ & 0.097 & 0 \\
\hline Ace inhibitors & $62.4 \%$ & $62.1 \%$ & 0.77 & 0.0066 & 0.006 \\
\hline Angiotensin receptor blockers & $33.8 \%$ & $33.8 \%$ & 0.98 & 0.0005 & 0.0222 \\
\hline Beta blockers & $85.7 \%$ & $85.4 \%$ & 0.74 & 0.0075 & 0.042 \\
\hline Calcium channel blockers & $59.6 \%$ & $57.3 \%$ & 0.03 & 0.0475 & 0.0179 \\
\hline Digoxin & $23.4 \%$ & $19.8 \%$ & $<.001$ & 0.0892 & 0.0403 \\
\hline Proton pump inhibitors & $56.2 \%$ & $49.5 \%$ & $<0.001$ & 0.1356 & 0.0753 \\
\hline NSAIDS & $53.2 \%$ & $48.7 \%$ & $<0.001$ & 0.091 & 0.133 \\
\hline Antiplatelets & $27.9 \%$ & $22.5 \%$ & $<0.001$ & 0.1229 & 0.0785 \\
\hline Insulin & $8.5 \%$ & $8.2 \%$ & 0.54 & 0.0136 & 0.0247 \\
\hline Statins & $75.4 \%$ & $75.9 \%$ & 0.6 & 0.0114 & 0.0608 \\
\hline \multicolumn{6}{|l|}{ Renal Disease } \\
\hline None or mild & $100.0 \%$ & $100.0 \%$ & & & $\begin{array}{l}\text { Not available } \\
\text { due to perfect } \\
\text { match }\end{array}$ \\
\hline
\end{tabular}

Moderate (GFR 30-60 ml/min/1.73 m2)

Severe

$($ GFR $<30 \mathrm{ml} / \mathrm{min} / 1.73 \mathrm{~m} 2)$

Abbreviations: $A C E$ Angiotensin converting enzyme, $A M I$ Acute myocardial infarction, COPD Chronic obstructive pulmonary disease, ICD International classification of diseases, NSAIDS Non-steroidal antiinflammatory drugs, P-GP P-glycoprotein, CYP Cytochrome

reductions only, and did not consider use of p-gp inhibitors or dual p-gp and CYP3A4 inhibitors in assessing dosing criteria.

In aggregate our findings demonstrate that decisions by healthcare providers about DOAC dosing may be based on patient clinical conditions not reflected in FDA dosing recommendations. Our analysis suggests that patients deemed by providers to be at higher bleeding risk may have received low dose DOACs even though FDA criteria suggest that they qualified for standard dose. It is noteworthy that our unadjusted analyses found higher risk of bleeding in patients who met criteria for standard dose rivaroxaban or dabigatran but received low dose, suggesting that the perception of higher bleeding risk by physicians was warranted, and may point to important patient characteristics not reflected in FDA criteria. Similarly, patients qualifying for low dose may have been prescribed standard doses if providers deemed their bleeding risk to be low.

DOAC-specific factors should predominantly affect dosing decisions. Renal function is the main indicator for low dose dabigatran and rivaroxaban. Nearly 80\% of ingested dabigatran is metabolized by the kidney, while $\sim 30 \%$ of rivaroxaban is metabolized by the kidney. With chronic kidney disease, the half-lives of these medications are extended, leading to potentially high plasma concentrations of the medications and increased bleeding risk [20, 21]. DOAC dose adjustments based on renal function therefore reflect the increased bleeding risk in patients with compromised renal function. Several drug interactions also affect dosing of DOACs. Dabigatran is a substrate for P-glycoprotein. Concomitant use of dabigatran with $\mathrm{P}$-gp inducers such as rifampin reduces the anticoagulant effect of dabigatran and should be avoided 


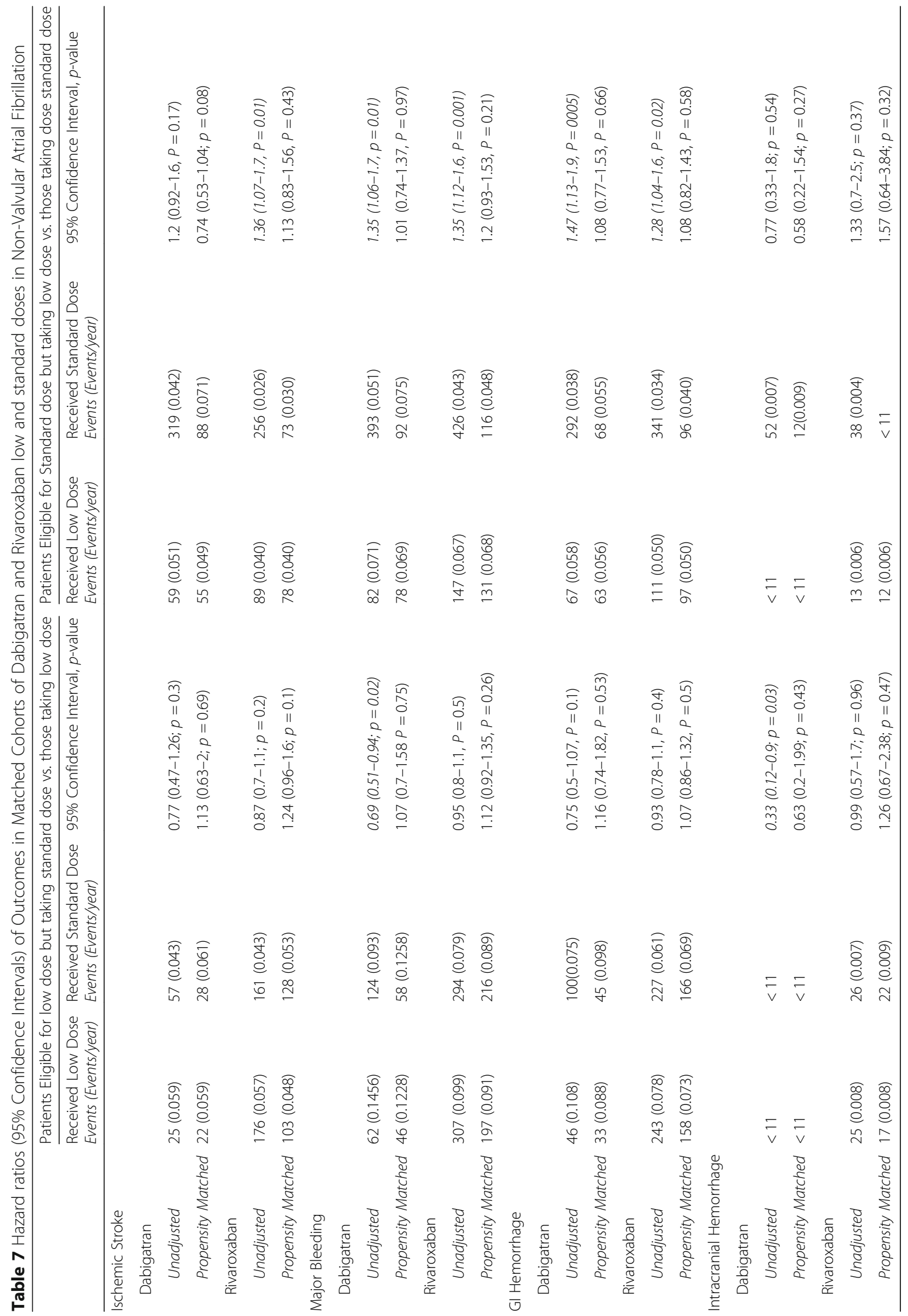


whereas use of dabigatran with P-gp inhibitors (eg, ketoconazole, dronedarone) in patients with renal disease may increase the anticoagulant effect, hence dose adjustment is required. Combined P-gp and CYP3A4 inhibitors (ketoconazole, protease inhibitors) increase the anticoagulant effects of rivaroxaban and should not be used concomitantly with rivaroxaban. In our analysis, we found a significant percentage of patients on medications interfering with the metabolism of dabigatran and rivaroxaban. Notably, receipt of standard dose dabigatran among patients qualifying for low dose dabigatran due to eGFR $30-60 \mathrm{ml} / \mathrm{min}$ with concomitant use of a p-gp inhibitor was particularly common, suggesting that some providers may not recognize the potential interaction. We also note, however, that our pharmacy data only permit evaluation of prescription fills, thus it is possible that some patients receiving concomitant p-gp inhibitors were instructed not to take them or switched to an alternative drug, in which case they would not meet criteria for low dose dabigatran. Other combinations that increase the risk of bleeding with DOACs are antiplatelet agents and non-steroidal anti-inflammatory drugs (NSAIDs), although no specific dosing adjustments are recommended for patients on these drugs. In our study, we found that use of prescription anti-platelet use among patients who met criteria for standard dose dabigatran or rivaroxaban was associated with a modestly higher use of low dose dabigatran and rivaroxaban. Similarly, use of low dose rivaroxaban was also more frequent among rivaroxaban patients taking NSAIDS.

\section{Limitations}

An important limitation of this paper is our inability to measure GFR using the Cockcroft-Gault [CG] equation, which is reflected in FDA recommendations for DOAC dosage reductions. There are three commonly used equations for estimating GFR: the oldest is the CG equation, originally published in 1976, followed by the MDRD, updated MDRD, and CKD-EPI equations in 1999, 2005, and 2009, respectively [15, 16, 22]. The MDRD and CKD-EPI equations use serum creatinine in combination with age, sex, and race to estimate GFR, while CG also uses patient weight. The use of alternative equations for dosing decisions has been the topic of considerable debate. In clinical practice, physicians rarely use the CG-estimated GFR and instead rely on the MDRD or CKD-EPI equations, which are easy to calculate and often automatically reported with serum creatinine laboratory tests. Notably, the National Kidney Disease Education Program (NKDEP) previously indicated that either the MDRD or CG equation may be used for drug dosing decisions [23], while more recently, the National Institute of Diabetes and Digestive and Kidney Diseases suggested that either CKD-EPI or CG equations are appropriate for drug dosing purpose [24]. In contrast, a previous review of FDA-recommended drug dosing showed that the CG equation is historically the most common renal function equation cited in drug dosing recommendations [25]. As noted by Yao et al. [26], it is likely that FDA drug labels historically relied on CG-estimated GFR because this method was available before the MDRD or CKD-EPI equations were developed and widely adopted, and drug dosage recommendations have not caught up to standard clinical practice with respect to assessing renal function. Nevertheless, this poses inconsistencies with clinical practice. While for most patients, GFR estimated by the MDRD or CKD-EPI equations has reasonable concordance with CG-estimated GFR, for older patients and patients with significant comorbidity, malnutrition leading to decreased muscle mass, or morbid obesity, there may be less agreement and dosing of medications based on the former may not be consistent with FDA recommendations [ 27-29]. Schwartz [30] found that use of the MDRD or CKD-EPI equations rather than the CG equation for estimating GFR may fail to identify 20 to $50 \%$ of patients for whom reduced dabigatran and rivaroxaban doses are recommended. Thus, our analysis likely underestimates the number of patients who qualify for low dose rivaroxaban and dabigatran if decisions are based on the CG equation, thereby underestimating the proportion of patients who are overdosed, or overestimating the proportion of patients who are underdosed, relative to FDA criteria. However, they do likely reflect dosing decisions based on GFR estimates typically used in clinical practice.

Other potential limitations of this study should also be considered. First, due to the observational nature of the study, it is possible that unmeasured confounders could have affected our results in spite of using propensity matched analysis. Second, our analysis included patients over the age of 65 and the findings require validation in younger patients. Finally, we lacked detailed evidence on AF burden and estimation of thromboembolic risk. Strengths our study are the large sample size, the availability of laboratory results (GFR) for most patients, incorporating concomitant medication use for assessing dosing criteria, and application of risk adjustment methods including propensity-matched analyses.

\section{Conclusion}

The purpose of our study was to improve understanding of safety and efficacy of DOACs in AF patients receiving low or standard dosing of these medications that was inconsistent with FDA criteria. The majority of patients qualifying for low dose DOACs received standard doses and a percentage of patients qualifying for standard dosing received low dose. After adjustment for comorbidities the 
risk of stroke and major bleeding was not affected by use of dose inconsistent with FDA criteria. Further validation of our results is warranted especially in patients at high thromboembolic or bleeding risk.

\section{Supplementary information}

Supplementary information accompanies this paper at https://doi.org/10. 1186/s12872-020-01340-4.

Additional file 1: Table S1. ICD 9 and 10 codes of comorbidities and outcomes included in the analysis and list of medications used for dose adjustments of dabigatran and rivaroxaban. Description of Data: All ICD 9 and 10 codes included in the analysis.

Additional file 2: Table S2. Hazard ratios (95\% Confidence Intervals) of Outcomes in Cohorts of Dabigatran and Rivaroxaban low and standard doses in Non-Valvular Atrial Fibrillation. Description of Data: Hazard ratios of Outcomes from the Analysis of dose adjustments based on renal function only.

\section{Abbreviations}

ACE: Angiotensin-converting-enzyme; AF: Atrial fibrillation; Cl: Confidence intervals; CKD: Chronic kidney disease; CrCL: Creatinine clearance); DOACs: Direct oral anticoagulants; eGFR: Estimated glomerular filtration rate; FDA: Food and Drug Administration; ICD-9-CM: International classification of diseases-ninth revision-clinical modification; ICH: Intracranial hemorrhage; MDRD: Modification of diet in renal disease; NKDEP: National Kidney Disease Education Program; P-gp: P-glycoprotein; P-gp-Cyp3A4: P-gp and cytochrome-3A4; RCT: Randomized controlled trials; RR: Relative rates; VKA: Vitamin K antagonist

\section{Acknowledgements}

None.

\section{Author disclosures}

The authors have no conflicts of interest to disclose.

\section{Authors' contributions}

AB drafted manuscript, MVS and YG did analysis and reviewed the final version of the manuscript, all other authors (Cl, RA, PA, EC, EL) reviewed manuscript and modified content. All authors have read and approved the final version of the manuscript.

\section{Funding}

None.

\section{Availability of data and material}

The data that support the findings of this study are available from PearlDiver, Inc. but restrictions apply to the availability of these data, which were used under license for the current study, and so are not publicly available. Data are however available from the authors upon reasonable request and with permission of PearlDiver, Inc. (info@pearldiverinc.com).

\section{Ethics approval and consent to participate}

All data used in this study were anonymized for use and accessed through a HIPAA-compliant platform maintained by PearlDiver, Inc.

\section{Consent for publication}

Not applicable, this not a case report.

\section{Competing interests}

Alexandros Briasoulis is a member of the editorial board of this journal.

\section{Author details}

'Division of Cardiovascular Diseases, Section of Heart Failure and Transplant, University of lowa College of Medicine, lowa City, IA, USA. ${ }^{2}$ Division of Cardiovascular Medicine, 200 Hawkins Dr, lowa City, IA 52242, USA. ${ }^{3}$ Division of General Medicine, University of lowa College of Medicine, lowa City, IA USA. ${ }^{4}$ Department of Epidemiology, University of lowa College of Public
Health, lowa City, IA, USA. ${ }^{5}$ Departments of Neurology and Neurosurgery, University of lowa College of Medicine, lowa City, USA. ${ }^{6}$ Comprehensive Access and Delivery Research and Evaluation Center, lowa City VA Medical Center, IA, 200 Hawkins Dr, lowa City, IA 52242, USA.

Received: 22 May 2019 Accepted: 13 January 2020

Published online: 03 February 2020

\section{References}

1. Wolf PA, Abbott RD, Kannel WB. Atrial fibrillation as an independent risk factor for stroke: the Framingham study. Stroke. 1991;22:983-8.

2. Friberg L, Rosenqvist M, Lip GY. Net clinical benefit of warfarin in patients with atrial fibrillation: a report from the Swedish atrial fibrillation cohort study. Circulation. 2012;125:2298-307.

3. Poli D, Antonucci E, Testa S, Tosetto A, Ageno W, Palareti G, Italian Federation of Anticoagulation C. Bleeding risk in very old patients on vitamin $\mathrm{K}$ antagonist treatment: results of a prospective collaborative study on elderly patients followed by Italian Centres for anticoagulation. Circulation 2011;124:824-829.

4. Connolly SJ, Ezekowitz MD, Yusuf S, Eikelboom J, Oldgren J, Parekh A, Pogue J, Reilly PA, Themeles E, Varrone J, Wang S, Alings M, Xavier D, Zhu J, Diaz R, Lewis BS, Darius H, Diener HC, Joyner CD, Wallentin L, Committee RLS, Investigators. Dabigatran versus warfarin in patients with atrial fibrillation. N Engl J Med 2009;361:1139-1151.

5. Patel MR, Mahaffey KW, Garg J, Pan G, Singer DE, Hacke W, Breithardt G, Halperin JL, Hankey GJ, Piccini JP, Becker RC, Nessel CC, Paolini JF, Berkowitz SD, Fox KA, Califf RM, Investigators RA. Rivaroxaban versus warfarin in nonvalvular atrial fibrillation. N Engl J Med. 2011;365:883-91.

6. Granger CB, Alexander JH, McMurray JJ, Lopes RD, Hylek EM, Hanna M, AlKhalidi HR, Ansell J, Atar D, Avezum A, Bahit MC, Diaz R, Easton JD, Ezekowitz JA, Flaker G, Garcia D, Geraldes M, Gersh BJ, Golitsyn S, Goto S, Hermosillo AG, Hohnloser SH, Horowitz J, Mohan P, Jansky P, Lewis BS, Lopez-Sendon JL, Pais P, Parkhomenko A, Verheugt FW, Zhu J, Wallentin L, Committees A, Investigators. Apixaban versus warfarin in patients with atrial fibrillation. N Engl J Med 2011:365:981-992.

7. Giugliano RP, Ruff CT, Braunwald E, Murphy SA, Wiviott SD, Halperin JL, Waldo AL, Ezekowitz MD, Weitz JI, Spinar J, Ruzyllo W, Ruda M, Koretsune Y, Betcher J, Shi M, Grip LT, Patel SP, Patel I, Hanyok JJ, Mercuri M, Antman EM, Investigators EA-T. Edoxaban versus warfarin in patients with atrial fibrillation. N Engl J Med. 2013;369:2093-104.

8. Lehr T, Haertter S, Liesenfeld KH, Staab A, Clemens A, Reilly PA, Friedman J. Dabigatran etexilate in atrial fibrillation patients with severe renal impairment: dose identification using pharmacokinetic modeling and simulation. J Clin Pharmacol. 2012:52:1373-8.

9. Martin JL, Esmaeili H, Manuel RC, Petrini M, Wiebe S, Maas H. Pharmacokinetics/ pharmacodynamics of Dabigatran 75 mg twice daily in patients with Nonvalvular atrial fibrillation and severely impaired renal function. J Cardiovasc Pharmacol Ther. 2018:23:399-406.

10. Camm AJ, Lip GY, De Caterina R, Savelieva I, Atar D, Hohnloser SH, Hindricks G, Kirchhof P, Guidelines ESCCFP. 2012 focused update of the ESC Guidelines for the management of atrial fibrillation: an update of the 2010 ESC Guidelines for the management of atrial fibrillation. Developed with the special contribution of the European Heart Rhythm Association. Eur Heart J 2012;33:2719-2747.

11. January CT, Wann LS, Alpert JS, Calkins H, Cigarroa JE, Cleveland JC Jr, Conti $J B$, Ellinor PT, Ezekowitz MD, Field ME, Murray KT, Sacco RL, Stevenson WG, Tchou PJ, Tracy CM, Yancy CW, American College of Cardiology/American Heart Association Task Force on Practice G. 2014 AHA/ACC/HRS guideline for the management of patients with atrial fibrillation: a report of the American College of Cardiology/American Heart Association task force on practice guidelines and the Heart Rhythm Society. J Am Coll Cardiol. 2014; 64:e1-76.

12. Basaran O, Filiz Basaran N, Cekic EG, Altun I, Dogan V, Mert GO, Mert KU, Akin F, Soylu MO, Memic Sancar K, Biteker M. PRescriptiOn PattERns of Oral anticoagulants in Nonvalvular atrial fibrillation (PROPER study). Clin Appl Thromb Hemost. 2017:23:384-91.

13. Sorensen R, Gislason G, Torp-Pedersen C, Olesen JB, Fosbol EL, Hvidtfeldt MW, Karasoy D, Lamberts M, Charlot M, Kober L, Weeke P, Lip GY, Hansen ML. Dabigatran use in Danish atrial fibrillation patients in 2011: a nationwide study. BMJ Open. 2013;3:e002758. https://doi.org/ 10.1136/bmjopen-2013- 002758. 
14. Steinberg BA, Shrader P, Thomas L, Ansell J, Fonarow GC, Gersh BJ, Kowey PR, Mahaffey KW, Naccarelli G, Reiffel J, Singer DE, Peterson ED, Piccini JP, Investigators O-A, Patients. Off-label dosing of non-vitamin K antagonist Oral anticoagulants and adverse outcomes: the ORBIT-AF II registry. J Am Coll Cardiol 2016;68:2597-2604.

15. Steinberg BA, Shrader P, Pieper K, Thomas L, Allen LA, Ansell J, Chan PS, Ezekowitz MD, Fonarow GC, Freeman JV, Gersh BJ, Kowey PR, Mahaffey KW, Naccarelli GV, Reiffel JA, Singer DE, Peterson ED, Piccini JP. Outcomes Registry for Better Informed Treatment of Atrial Fibrillation III. Frequency and Outcomes of Reduced Dose Non-Vitamin K Antagonist Anticoagulants: Results From ORBIT-AF II (The Outcomes Registry for Better Informed Treatment of Atrial Fibrillation II). J Am Heart Assoc. 2018;7(4). https://doi. org/10.1161/JAHA.117.007633.

16. Levey AS, Coresh J, Greene T, et al. Using standardized serum creatinine values in the modification of diet in renal disease study equation for estimating glomerular filtration rate. Ann Intern Med. 2006;145(4):247-54.

17. Levey AS, Stevens LA, Schmid CH, et al. A new equation to estimate glomerular filtration rate. Ann Intern Med. 2009;150(9):604-12.

18. Charlson M, Szatrowski TP, Peterson J, Gold J. Validation of a combined comorbidity index. J Clin Epidemiol. 1994;47:1245-51.

19. Yao X, Shah ND, Sangaralingham LR, Gersh BJ, Noseworthy PA. Non-vitamin $\mathrm{K}$ antagonist oral anticoagulant dosing in patients with atrial fibrillation and renal dysfunction. J Am Coll Cardiol. 2017;69:2779-90.

20. Stangier J, Rathgen K, Stahle H, Mazur D. Influence of renal impairment on the pharmacokinetics and pharmacodynamics of oral dabigatran etexilate: an open-label, parallel-group, single-Centre study. Clin Pharmacokinet. 2010; 49:259-68.

21. Food and Drug Administration. Guidance for industry: Pharmacokinetics in patients with impaired renal function - Study design, data analysis, and impact on dosing and labeling. US Department of Health and Human Services, Rockville MD 2010. https://www.fda.gov/downloads/drugs/ guidances/ucm204959.pdf)

22. Cockcroft DW, Gault MH. Prediction of creatinine clearance from serum creatinine. Nephron. 1976;16(1):31-41.

23. Health professionals CKD and drug dosing information for providers. Estimation of kidney function for prescription medication dosage in adults. http://www.nkdep.nih.gov/professionals/drug-dosinginformation.htm. Accessed 17 Jan 2019.

24. The National Institute of Diabetes and Digestive and Kidney Diseases. Estimation of Kidney Function for Prescription Medication Dosage in Adults. 2015. Available: http://www.niddk.nih.gov/health-information/healthcommunication-programs/nkdep/a-z/ckd-drug-dosing/Pages/CKD-drugdosing.aspx. Accessed 17 Jan 2019.

25. Dowling TC, Matzke GR, Murphy JE, Burckart GJ. Evaluation of renal drug dosing: prescribing information and clinical pharmacist approaches. Pharmacotherapy. 2010;30(8):776-86.

26. Yao X, Shah ND, Sangaralingham LR, Gersh BJ, Noseworthy PA. Reply: NOAC dosing in patients with atrial fibrillation and renal dysfunction. J Am Coll Cardiol. 2017;70(21):2734-5.

27. Dowling TC, Wang ES, Ferrucci L, Sorkin JD. Glomerular filtration rate equations overestimate creatinine clearance in older individuals enrolled in the Baltimore longitudinal study on aging: impact on renal drug dosing. Pharmacotherapy. 2013;33:912-21.

28. Park EJ, Wu K, Mi Z. A systematic comparison of the Cockcroft-Gault and modification of diet in renal disease equations for classification of kidney dysfunction and dosage adjustment. Ann Pharmacother. 2012;46:1174-87.

29. Rule AD, Larson TS, Bergstralh EJ, Slezak JM, Jacobsen SJ, Cosio FG. Using serum creatinine to estimate glomerular filtration rate: accuracy in good health and in chronic kidney disease. Ann Intern Med. 2004;141(12):929-37. https://doi.org/10.7326/0003-4819-141-12-200412210-00009.

30. Schwartz J. Potential impact of substituting estimated glomerular filtration rate for estimated creatinine clearance for dosing of direct oral anticoagulants. J Am Geriatr Soc. 2016;64:1996-2002.

\section{Publisher's Note}

Springer Nature remains neutral with regard to jurisdictional claims in published maps and institutional affiliations.

Ready to submit your research? Choose BMC and benefit from:

- fast, convenient online submission

- thorough peer review by experienced researchers in your field

- rapid publication on acceptance

- support for research data, including large and complex data types

- gold Open Access which fosters wider collaboration and increased citations

- maximum visibility for your research: over $100 \mathrm{M}$ website views per year

At BMC, research is always in progress.

Learn more biomedcentral.com/submissions 